\title{
34. Die koptischen Paginae von P.Yale Inv. 1804. Mit einem Anhang zu den koptischen Pachturkunden
}

\author{
Sebastian Richter (Leipzig)
}

\begin{abstract}
Reedition of the Coptic pages of the documentary codex P.Yale Inv. 1804 (editio princeps by MacCoull 1986). The codex, composed of Greek and Coptic documents, apparently originates from Antinoupolis, and seems to be centered around the monastery of Apa Surus. Improved readings allow identification of document types. Apart from lists and accounts, the most frequently attested type is the misthosis land lease. Fragments of more than ten land leases are preserved, shedding new light on the Coptic lease. An appendix is attached, presenting basic information on provenances, dates, formularies, clauses and key terms of Coptic lease documents.
\end{abstract}

Keywords: Coptic papyri, leases, monastery, economy, codex (documentary)

Mitte der 90er Jahre veröffentlichten Ruth Duttenhöfer und Klaas Worp unter dem Titel „Die griechischen Paginae von P.Yale inv. 104“ eine partielle Neuedition des dokumentarischen Papyruskodex inv. 1804 der Papyrus Collection der Beinecke Rare Book and Manuscript Library, ${ }^{1}$ der zuvor im Ganzen durch Leslie MacCoull der Fachwelt bekannt und in einer ersten Edition zugänglich gemacht worden war. ${ }^{2}$ Die in dieser Akte zusammengefassten Dokumente legen in den beiden Sprachen Griechisch und Koptisch Zeugnis von wirtschaftlichen Transaktionen ab, in deren Zentrum das Kloster des Apa Surus in Antinoupolis zu stehen scheint. Der Erhaltungszustand der Kodexseiten ist schlecht und die Lesung der einzelnen Blätter zumeist recht mühsam, so dass die editio princeps zu einigen Fehlschlüssen kam. Zu diesen falschen Annahmen gehörte neben der Herkunft des Kode $^{3}$ und der kodikologischen Rekonstruktion der Blattfolge auch das ursprüngliche Format der Kodexseiten, das in der editio princeps zu $16 \mathrm{~cm}$ Höhe bei $14 \mathrm{~cm}$ Breite rekonstruiert ist und mit einem frühen square Typ ${ }^{4}$ verglichen wird. Diese Maße entsprechen den materialiter tatsächlich erreichten maximalen Höhen und Breiten etlicher Seiten; MacCoull ging also von in dieser Beziehung mehr

${ }^{1}$ Ruth Duttenhöfer \& Klaas A. Worp „Die griechischen Paginae von P.Yale inv. 1804“. Tyche 11 (1996), 97-106.

${ }^{2}$ Leslie S.B. MacCoull, Coptic Documentary Papyri in the Beinecke Library, Yale University. Textes et documents, tome 17. Cairo 1986 (= P.Yale copt.).

${ }^{3}$ MacCoull vermutete Aphrodito.

${ }^{4}$ Turner, The early Codex, group 9, p. 21 ,(,Square', Schubart, e.g. 15 x 17, 14 x 16 or 13/12 x 15/13)“". 
oder weniger vollständigen Blättern aus. Indessen belegt die von MacCoull zusammengestellte Übersicht dokumentarischer Codices des 6.-8. Jhs. den zeitgenössischen Usus von Hochformaten, namentlich von Blatthöhen um $30 \mathrm{~cm},{ }^{5}$ und auch der dokumentarische Kodex P.Yale inv. 1804 dürfte in dieser Hinsicht keine Ausnahme gebildet haben. Die nach verbesserter Lesung deutlicher hervortretenden Urkundenformulare zeigen klar, dass nicht eine einzige Seite ohne erheblichen Textverlust überkommen ist. Damit vergrößert sich die Problematik des Kodex leider beträchtlich. Zu dem Umstand, dass wir mit unvollständigen Dokumenten vorlieb nehmen müssen, ist noch folgendes sonderbare Phänomen zu konstatieren: Wir haben eine ganze Reihe von Urkundenanfängen und etwa ebenso viele Urkundenschlüsse, jedoch keinen einzigen Join, etwa so, als sei das ursprüngliche Buch der Länge und Breite nach halbiert worden, und wir hätten nun z.B. das vordere obere und das hintere untere Viertel in den Händen.

Während die kodikologischen Fragen hier nur aufgeworfen und als weiter anstehende Aufgabe annnonciert werden können, sollen die folgenden Zeilen in erster Linie das koptologische Komplement zu Duttenhöfer \& Worp 1996 liefern, ${ }^{6}$ die Neuedition der koptischen Seiten des Kodex.

Die Neuedition der griechischen paginae hatte zur Identifikation mehrerer griechischer Misthosis-Urkunden geführt. ${ }^{7}$ Diese Geschäftsart hatte sich bereits in der unvollkommenen Lesung der editio princeps auch für mehrere der koptischen paginae abgezeichnet. ${ }^{8}$ Durch die hier vorgelegte Ausbeute von zwei Gelegenheiten ${ }^{9}$ zur Kollation der Texte wird dieser Eindruck vollauf bestätigt. Da sich der Honorandus dieser Festschrift, wie in so vielem anderen, auch als Kenner der spätantiken Misthosis ausgezeichnet hat, da jedoch das Dossier der koptischen Urkunden zur Pacht bislang so gut wie unbekannt geblieben ist, ${ }^{10}$ ergreife ich die Gelegenheit zu einem provisorischen Überblick über dieses entlegene, jedoch keineswegs unergiebige Feld der papyrologischen Dokumentation (s.u. den Anhang).

\footnotetext{
${ }^{5}$ MacCoull, op. cit., 2-4.
}

${ }^{6}$ Was von Duttenhöfer \& Worp 1996, 97, bereits angeregt worden ist: „Leider sind wir nicht in der Lage, auch die koptisch geschriebenen Teile des Kodex vollständig zu interpretieren. Diese Aufgaben müssen den Koptologen vorbehalten bleiben."

${ }^{7}$ Fol. 22v, fol. 22r, fol. 23r nach der Seitenzählung der ed. princ.

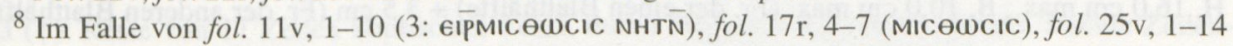

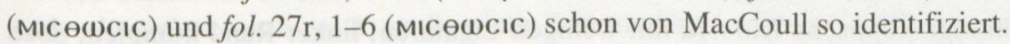

${ }^{9}$ Im Juni 2005 und im Juli 2007 jeweils am Rande von Editorial Meetings im Rahmen des Projekts Critical Edition of the Complete Works of Shenoute. Mein Dank gilt Prof. Andrew Crislip, Prof. Stephen Emmel und Prof. Bentley Layton für die glänzende Organisation dieser Treffen und die Einladung dazu!

${ }^{10}$ Die Aufarbeitung dieses Dossiers, die ich 2005 in meiner Habilitationsschrift „Pacht nach koptischen Quellen. Beiträge zur Rechts-, Wirtschafts- und Sozialgeschichte des byzantinischen und früharabischen Ägypten" unternommen habe, soll in der Reihe Papyrologica Vindobonensia publiziert werden. Zur Terminologie der koptischen Miet- und Pachturkunden vgl. T.S. Richter, „Alte Isoglossen im Rechtswortschatz koptischer Urkunden,“ Lingua Agyptia 10 (2002), 389-399 und id., „Koptische Mietverträge über Gebäude und Teile von Gebäuden“, JJP 32 (2002), 113168 . 
Die hier vorgelegte Neuedition berschränkt sich auf Seiten, bei denen die Kollation zu nennenswerten Korrekturen oder Textzuwachs geführt hat. Die zur Identifikation verwendeten Sigla A1-17 und B1-14 zitieren die Shelfmarks, unter denen die einzeln verglasten Blätter von Inv. 1804 in der Beinecke Rare Book and Manuscript Library geführt werden, da die in der ed. princ. eingeführte FolioZählung, wie ich fürchte, nicht die ursprüngliche Seitenfolge repräsentiert und insofern irreführend ist. ${ }^{11}$ Die Unterschiede zwischen den in der Neuedition vorgeschlagenen Lesungen und dem Text der ed. princ. werden nicht eigens angezeigt.

\section{A2 (Ed. pr. p. 19, fol. 2r/v): Abrechnung für den Monat Athyr}

H. $15,5 \mathrm{~cm}$ max.; B. 16,0 cm max.

A2 ( $\uparrow$ ): Links und unten ( $8 \mathrm{~cm}$ Rand) komplett, rechts und oben abgebrochen.

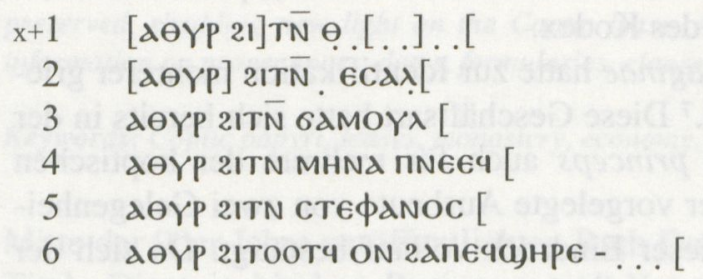

(Spatium $8 \mathrm{~cm}$ )

A2 $(\rightarrow)$ : Im oberen Teil Reste einer Zeile.

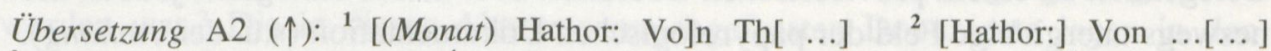
${ }^{3}$ Hathor: Von Gamûl [... ] ${ }^{4}$ Hathor: Von Mena, dem Schiffer [... ] ${ }^{5}$ Hathor: Von Stephanos [... $]{ }^{6}$ Hathor: Wieder von ihm, für seinen Sohn $[\ldots]$

\section{A3 (Ed. pr. p. 19-20, fol. 3r/v): Schluss einer Asphaleia $(\rightarrow)$ und Schluss einer weiteren Urkunde $(\uparrow)$}

H. 16,0 cm max.; B. 10,0 cm max. (Fr. der einen Blatthälfte) $+3,5 \mathrm{~cm}$ (Fr. der anderen Blatthälfte) A3 $(\rightarrow) 10$ Zeilen flüssige koptische Kursive mit wenigen Ligaturen; linker Rand komplett, doch Ausbruch am Zeilenanfang außer bei Z. 4-5; Text unten wohl nahezu vollständig; sonst abgebrochen. Am linken Rand Anschluss an den rechten Rand der anderen Blatthälfte. Ende der KyriaKlausel, Eid, Stipulation des Ausstellers, Zeugenunterschriften und Kompletionsvermerk.

11 Für deren Rekonstruktion könnten sich unter Umständen Muster von Wurmfraßspuren als hilfreich erweisen. 


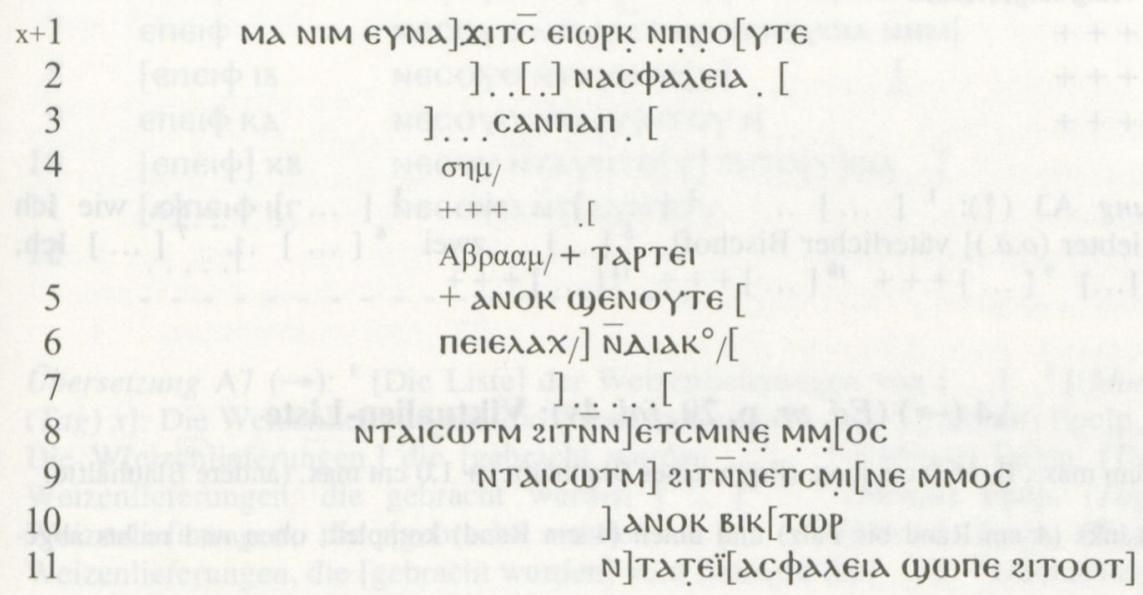

Übersetzung A3 $(\rightarrow):{ }^{1}$ [(sie ist gültig o.ä.) an jedem Ort, an den] sie genommen werden wird. Ich schwöre bei Go[tt ... ${ }^{2}[\ldots]$ Sicherheitsurkunde $[\ldots]^{3}[\ldots] \ldots[\ldots]$ ${ }^{4}[\ldots]$ Handzeichen des Abraham: $+++\ldots[\ldots]{ }^{5}$ (Zeugen:) + Ich, Schenute [... ] ${ }^{6}[\ldots$ der niedrigste $]$ Diakon, $[\ldots]{ }^{7}[\ldots] \ldots[\ldots]{ }^{8}[\ldots$ die ich gehört habe durch die $]$ sie Ausstellenden [ ... ] ${ }^{9}$ [... die ich gehört habe] durch die sie Ausstellenden ... ] ${ }^{10}$ Ich, Vik[tor, ... ${ }^{11}$ [Ich, NN, durch mich] ist diese [Sicherheitsurkunde entstanden.]

1 Koptische $\pi \alpha v \tau \alpha \chi 0 \hat{-}\left[\mathrm{Md}\right.$ NIM]-Klausel mit $x_{1}$ (statt $\mathrm{EM \phi aNIz \epsilon}$ etc.) z.B. in $P . K R U$ 97,81-82, jedoch fehlt hier der pronominale Rückbezug (vgl. P.KRU 97,81-82: 6РОq), daher ist die Ergänzung nicht sicher. Auch weisen die Misthosis-Urkunden aus Aschmunein diese Klausel gewöhnlich nicht auf (für die Mieturkunden vgl. Richter, JJP 2002, 156, n. 91).

5 Die Zeugenformel ist „Ich, $N N$, bin Zeuge für diese Asphaleia, die ich durch die sie Ausstellenden gehört habe", wie typisch für koptische Dokumente aus Antinou und aus der Aschmunein-Region, vgl. etwa CPR IV 49 passim, CPR IV 87,16 u.ö. Auch die griechischen Misthosis-Fragmente aus P.Yale inv. 1804 folgen diesem Formular, z.B.

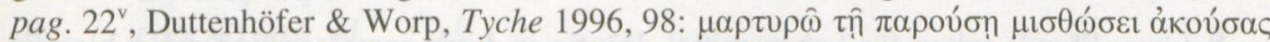

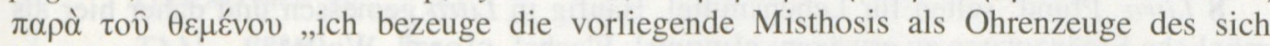
Verpflichtenden“. Vgl. noch hier A16 $(\rightarrow)$, B7 $(\rightarrow)$, B7 $(\uparrow)$, B9 $(\uparrow)$ und B13 $(\uparrow)$.

A3 ( $\uparrow)$ : Reste von 11 Zeilen koptischen Texts, durch diagonale Striche devaluiert. Rechte Zeilenenden komplett; nach ca. $5 \mathrm{~cm}$ Spatium Zeilenanfänge von ca. 10 Zeilen griechisch? - nur ca. 1 Buchstabe pro Zeile erhalten).

\begin{tabular}{|c|c|}
\hline 1 & $\ldots \ldots \epsilon$ \\
\hline 2 & NMOC \\
\hline 3 & $\lambda \bar{N} \Theta \epsilon \bar{N} T \Delta I$ \\
\hline 4 MEPIT & ] $\bar{N} \in I \omega^{T} N \in \Pi I C K^{\circ}$ \\
\hline 5 & . PO ECYNTE \\
\hline 6 & $\Delta l \ldots \ldots T$ \\
\hline 7 & + ANOK TEPKE \\
\hline
\end{tabular}


$\begin{array}{ll}8 & \text { ] völlig ausgebrochen } \\ 9 & \text { ] }+++ \\ 10 & ]+++ \\ 11 & ]+++\end{array}$

Übersetzung A3 $(\uparrow):{ }^{1}[\ldots] \ldots{ }^{2}[\ldots] \ldots$ sie ${ }^{3}[\ldots] \quad \ldots$ so, wie ich ${ }^{4}[\ldots$ geliebter $($ o. ä.) $]$ väterlicher Bischof $]{ }^{5}[\ldots] \ldots$ zwei ${ }^{6}[\ldots] \ldots{ }^{7}[\ldots]$ Ich, Terke, ${ }^{8}[\ldots]{ }^{9}[\ldots]+++{ }^{10}[\ldots]+++{ }^{11}[\ldots]+++$

\section{A4 $(\rightarrow)($ Ed. pr. p. 20, fol. 4v): Viktualien-Liste}

H. 14,5 cm max.; B. 16,0 cm max. (Frgm. einer Blatthälfte) +1,0 cm max. (andere Blatthälfte) A4 $(\rightarrow$ ): Links (4 cm Rand bis Falz) und unten (4 cm Rand) komplett; oben und rechts abgebrochen.

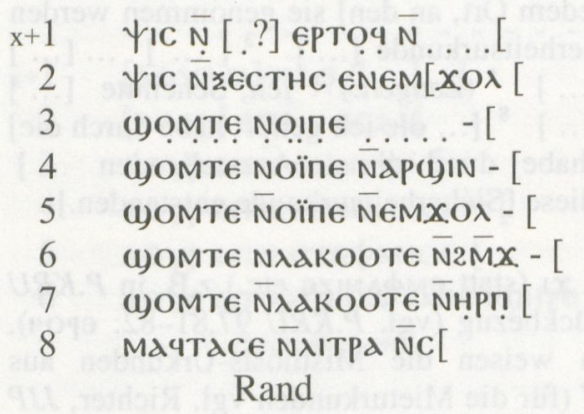

Übersetzung A4 $(\rightarrow):{ }^{1}$ Neun Artaben ... [ . . ] ${ }^{2}$ Neun Xesten Zwie[beln ...] ${ }^{3}$ Drei Oipen [... ${ }^{4}$ Drei Oipen Linsen [ ...] ${ }^{5}$ Drei Oipen Zwiebeln [...] ${ }^{6}$ Drei Lakoote $(=$ Knidien $)$ Essig $[\ldots] \quad{ }^{7}$ Drei Lakoote $(=$ Knidien $)$ Wein $[\ldots]$ ${ }^{8}$ Sechsunddreißig Litra S[ ... ]

8 Litra ,Pfund' selten für Lebensmittel. Häufig in Litra gemessen und daher hier als mögliche Ergänzungen zu erwägen: $\mathbf{c}[\mathrm{Inn \in N}]$,Flachs', c[OPT], Wolle'.

\section{$\mathrm{A} 7(\rightarrow)($ Ed. pr. p. 21, fol. 7v): \\ Liste über Weizenlieferungen im Monat Epep}

H. 15,5 cm max.; B. 16,0 cm max.

A7 $(\rightarrow)$ : Links ab Z. 5 und wohl auch oben(?) komplett; rechts und unten abgebrochen. Z. 8 und 10 Anfang jeweils $\rightarrow$ Faser abgeplatzt.

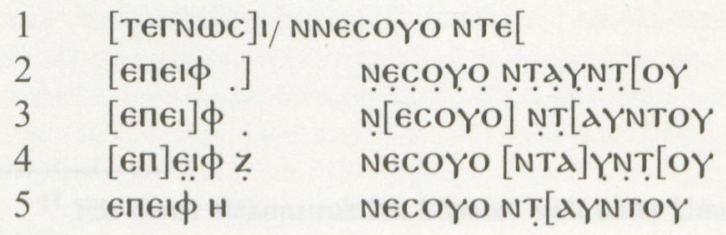




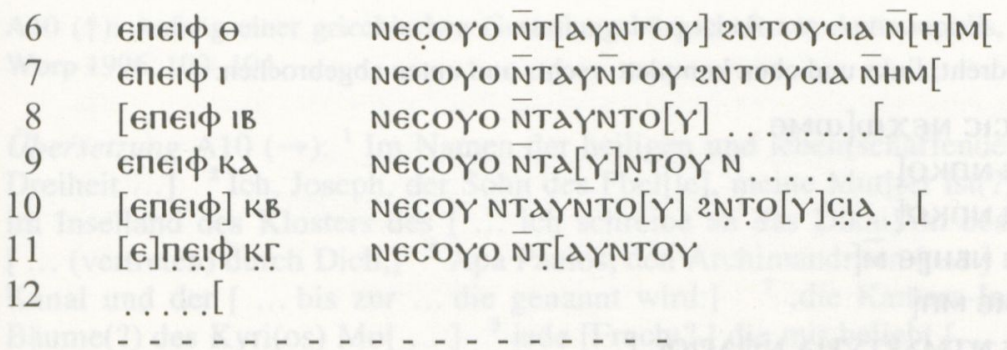

Übersetzung A7 $(\rightarrow):{ }^{1}$ [Die Liste] der Weizenlieferungen von [ ... ${ }^{2}{ }^{2}$ [(Monat) Epep, (Tag) $x]$ : Die Weizenlieferungen, die gebracht wurden $[\ldots]{ }^{3}[($ Monat $)$ Epe $] p$, (Tag) .... Die W[eizenlieferungen,] die [gebracht wurden ... ${ }^{4}{ }^{4}[($ Monat $)$ Ep]ep, (Tag) 7: Die Weizenlieferungen, die gebracht wurden [ ... ${ }^{5}$ (Monat) Epep, (Tag) 8: Die Weizenlieferungen, die [gebracht wurden ...] ${ }^{6}$ (Monat) Epep, (Tag) 9: Die Weizenlieferungen, die [gebracht wurden] vom Landgut Em[ ... $]^{7}$ (Monat) Epep, (Tag) 10: Die Weizenlieferungen, die [gebracht wurden] vom Landgut Em[ ... ] ${ }^{8}$ (Monat) Epep, (Tag) 11: Die Weizenlieferungen, die gebracht wurden [ ... $]{ }^{9}$ (Monat) Epep, (Tag) 21: Die Weizenlieferungen, die gebracht wurden $[\ldots]{ }^{10}$ (Monat) Epep, (Tag) 22: Die Weizenlieferungen, die [gebracht wurden] vom Landgut [ ... ] ${ }^{11}$ (Monat) Epep, (Tag) 23: Die Weizenlieferungen, die [gebracht wurden ... ] ${ }^{12} \ldots[\ldots]$

\section{A8 (Ed. pr. p. 21, fol. 8r/v): Viktualienliste und Bücherliste}

H. 15,0 cm max.; B. $16.0+3,0 \mathrm{~cm}$ (andere Doppelblatthälfte)

A8 $(\rightarrow)$ : Links (3,5 cm Rand) und unten ( $2 \mathrm{~cm}$ Rand) komplett; rechts und unten abgebrochen.

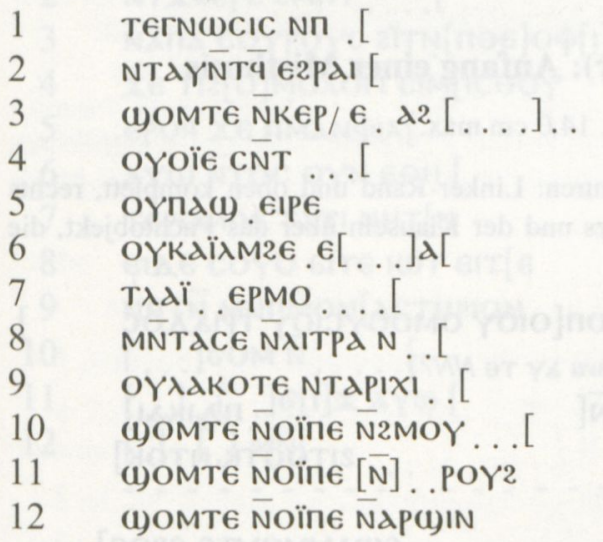

Übersetzung A8 $(\rightarrow)$ : ${ }^{1}$ Die Liste des [ ... ${ }^{2}$ der herbeigebracht wurde [... ] ${ }^{3}$ Drei Karat ... [ ... $]^{4}$ Zwei ... [ ... $]^{5}$ Ein Halb- $[\ldots]{ }^{6} \ldots[\ldots]{ }^{7} \ldots[\ldots]$

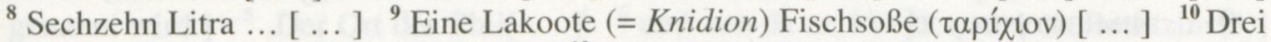
Oipen Salz ... [ ... ] ${ }^{11}$ Drei Oipen ... ${ }^{12}$ Drei Oipen Linsen ... [ ... ]

4 Nach der Form des Zahlworts ein feminines Objekt.

5 Ergänze evtl. [Md] 
A8 ( $\uparrow)$ : Um $180^{\circ}$ gedreht; links und oben komplett; rechts und unten abgebrochen.

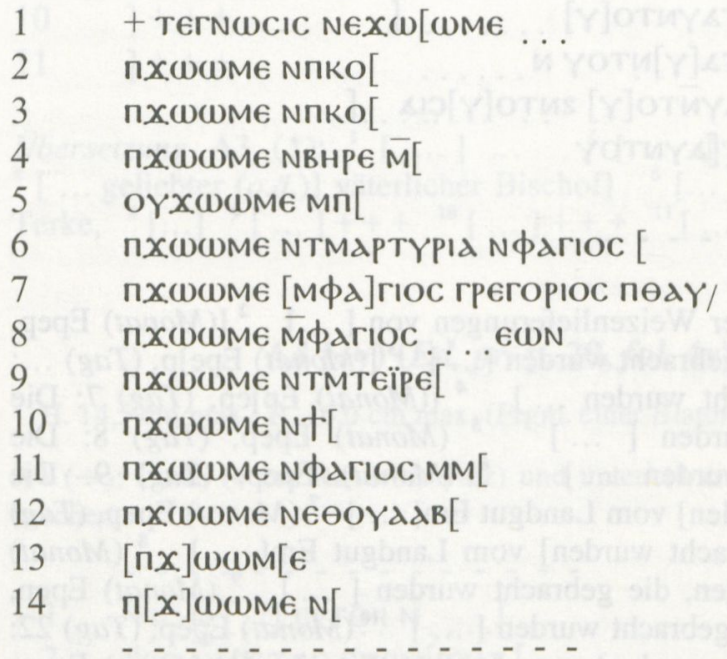

Übersetzung A8 ( $\uparrow):{ }^{1}$ Die Liste der Bü[cher ... ${ }^{2}$ Das Buch des ... [... $]{ }^{3}$ Das Buch des ... [ ... ${ }^{4}$ Das neue Buch des $[\ldots]{ }^{5}$ Ein Buch des $[\ldots]{ }^{6}$ Das Buch der Marter des Heiligen $[\ldots]{ }^{7}$ Das Buch des Heiligen Gregorios, des Wundertäters [ ... $]{ }^{8}$ Das Buch des Heiligen .... eon $[\ldots]{ }^{9}$ Das Buch der ... [ . . ] ${ }^{10}$ Das Buch der $[\ldots]{ }^{11}$ Das Buch des Heiligen M[ ... ${ }^{12}$ Das Buch der Heiligen $\left[\ldots[\ldots]{ }^{13}\right.$ Das B]uch [ ... ${ }^{12}$ Das [B] uch de[s ... ]

\section{$\operatorname{A10}(\rightarrow)(E d . p r$. p. 23, fol. 10r): Anfang einer Misthosis}

H. 14,5 cm max.; B. 14,0 cm max.

A10 $(\rightarrow)$ : Schrift nach rechts geneigt, einige Ligaturen: Linker Rand und oben komplett, rechts und unten abgebrochen. Teile des Anfangsformulars und der Klauseln über das Pachtobjekt, die Pachtdauer und den Pachtbeginn.

$\mathrm{x}+1$ f en ONOMATI THC ATIAC KaI [z] WOח[OIOY OMOOYCIOY TPIAAOC ]

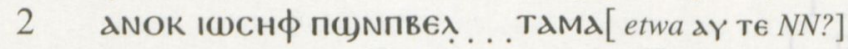

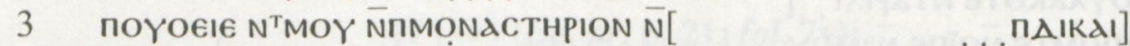

4 ON NIM[O]NACTHPION ETOY[AגB

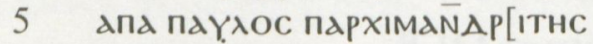

$6 \quad$ XIN nEYEIN NTTCO MNTTN [

$7 \quad x \in$ TMOY NKAMAP[

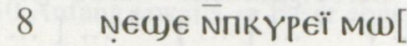

9 NIM EIOY $\omega(1) x[$

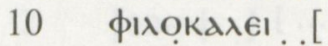

11 aүए NTOC O[N 
A10 $(\uparrow)$ : Anfang einer griechischen Gestellungsbürgschaft aus Antinoupolis, vgl. Duttenhöfer \& Worp 1996, 103-104.

Übersetzung A10 $(\rightarrow):{ }^{1}$ Im Namen der heiligen und leben[schaffenden, wesensgleichen Dreiheit ... $\quad{ }^{2}$ Ich, Joseph, der Sohn des Pbel[le], meine Mut[ter ist(?)... ] ${ }^{3}$ der Bauer im Inselland des Klosters des [ ... ich schreibe an das Dikai $]^{4}$ on des heiligen Klosters [ ... (vertreten) durch Dich,] ${ }^{5}$ Apa Paulos, den Archimandriten [ ... ] ${ }^{6}$ von dem TränkKanal und $\operatorname{der}\left[\ldots \text { bis zur ... die genannt wird:] }{ }^{7} \text {, die Kamara-Insel' [ ... }\right]^{{ }^{8}}$ der Bäume(?) des Kyri(os) Mo[ ... ${ }^{9}$ jede [Frucht?,] die mir beliebt [ ... ${ }^{10}$ instandhalten $[\ldots]^{11}$ und von da an [fort ... ]

2 Zur Ergänzung des Vatersnamens vgl. Hasitzka, Namenbuch, 67. - Ergänzung тамд[aY тє NN] nicht sehr wahrscheinlich, da die Erwähnung des Mutternamens in einer Misthosis kaum zu erwarten und in den koptischen Texten auch nicht belegt ist.

6 є()дүмоүтє: Oder, wie in Urkunden aus Aschmunein auch häufig, eүмоүтє.

9 Hier evtl. eine Variante der Wendung кдpпос NIM єıOүגay 4 der Pachturkunden aus Aschmunein (z.B. BKU III 347,7; CPR IV 117,20 u.ö.).

\title{
A11 (Ed.pr. p. 24, fol. 11r/v): Jeweils Anfang einer Misthosis ( $\uparrow$ und $\rightarrow$ )
}

\author{
H. 14,0 cm max.; B. 14,0 cm max.
}

A11 ( $\uparrow)$ : Gemäßigte Kursive ähnlich A10. Linker und oberer Rand komplett, rechts und unten abgebrochen. Teile des Anfangsformulars, der Pachterklärung und der Klauseln über das Pachtobjekt, die Pachtdauer, den Pachtzins und die Pächterarbeiten.

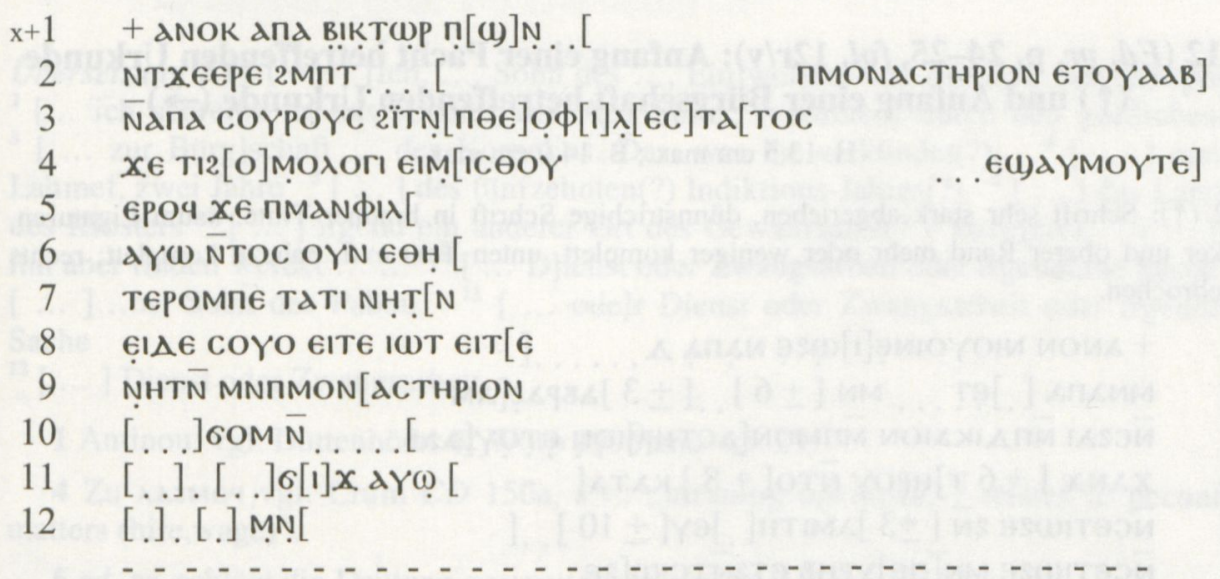

Übersetzung A11 $(\uparrow):{ }^{1}$ Ich, Apa Viktor, Sohn des $[\ldots, \text { aus ... }]^{2}$ der Tenne im [ ..., ich schreibe an das Dikaion des heiligen Klosters ] ${ }^{3}$ des Apa Surus, (vertreten) durch [den überaus g]ottliebenden ... ${ }^{4}$ Ich bekenne, dass ich pa[chte den ..., der genannt wird:] ${ }^{5}$,Der Ort des Phil[ ... ] ${ }^{e}{ }^{6}$ und von da an fort [ ... und als Pachtzins(?)] ${ }^{7}$ jährlich gebe ich euch [ ... $]^{\mathbf{8}}$ sei es Weizen, sei es Gerste, sei es [ ... ${ }^{9}{ }^{9}$ für euch und das Klo[ster ... $]^{10} \ldots$ Kraft ... $[\ldots] \quad{ }^{11} \ldots$ Hand und $[\ldots] \quad 12 \ldots$ und $[\ldots]$

4 є)думоүтє: Oder, wie in Urkunden aus Aschmunein auch häufig, єүмоүтє. 
A11 $(\rightarrow)$ : Breite Schrift, fast schräge Unziale; oberer und rechter Rand wohl komplett; linker Rand ausgefasert, unten abgebrochen. Teile des Anfangsformulars, der Pachterklärung und der Klauseln über das Pachtobjekt, die Pachtdauer, den Pachtzins und die Pächterarbeiten.

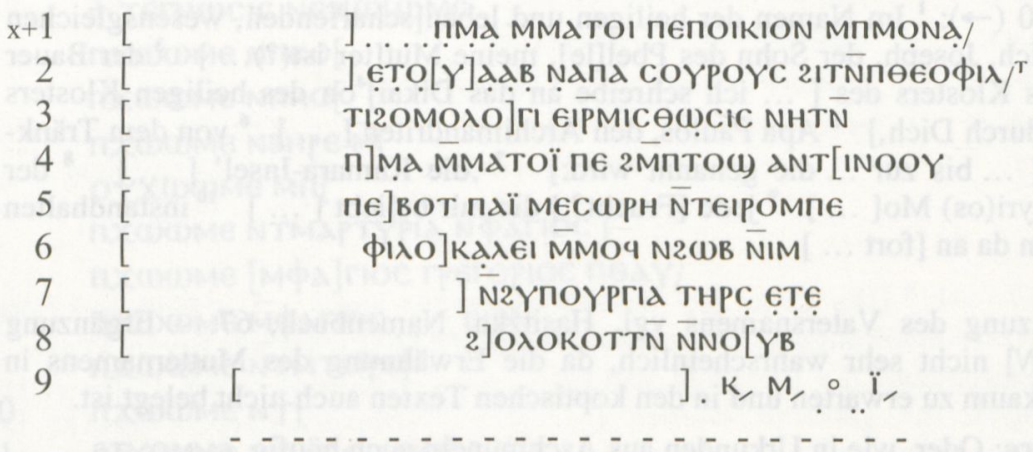

Übersetzung A11 $(\rightarrow):{ }^{1}$ [Ich, ..., Sohn des ... aus P]mam-matoi, dem Gehöft des heiligen Klosters ${ }^{2}$ [... ich schreibe an das Kloster] des Apa Surus, (vertreten) durch den überaus gottliebenden ${ }^{3}$ [ ... Ich beken]ne, dass ich euch eine Pachturkunde ausstelle ${ }^{4}$ [ ... P] mam-matoi ist es im Bezirk von Ant[inou ${ }^{5}[\ldots]$ diesem Monat Mesore dieses [Indiktions-]Jahres ${ }^{6}[\ldots]$ ihn betreuen in jeder Sache ${ }^{7}[\ldots]$ den geamten Dienst, nämlich ${ }^{8}[\ldots]$ Gold-Holokottinos ${ }^{9}[\ldots] \ldots$

9 In dieser Zeile sind Fasern verrutscht bzw. werden Teile der Zeile von verrutschten Fragmenten überdeckt.

\section{A12 (Ed. pr. p. 24-25, fol. 12r/v): Anfang einer Pacht betreffenden Urkunde $(\uparrow)$ und Anfang einer Bürgschaft betreffenden Urkunde $(\rightarrow)$}

H. 13,5 cm max.; B. 14,0 cm max.

A12 $(\uparrow)$ : Schrift sehr stark abgerieben, dünnstrichige Schrift in brauner Tinte, kaum Ligaturen. Linker und oberer Rand mehr oder weniger komplett, unten Text evtl. nahezu komplett; rechts abgebrochen.

\begin{tabular}{|c|c|}
\hline$x+1$ & 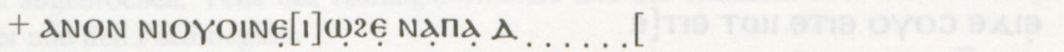 \\
\hline 2 & 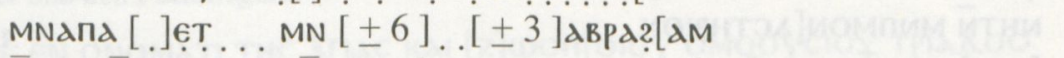 \\
\hline 3 & $\bar{N} C 2 d I \bar{N} \Pi \Delta I K d I O N$ MTMON[ACTHP]ION ETTOY $[$ ddB \\
\hline 4 & 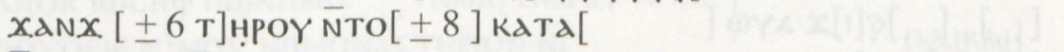 \\
\hline 5 & 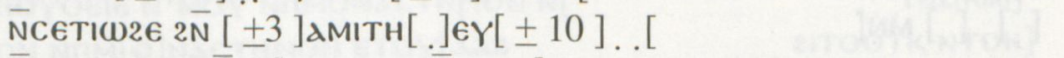 \\
\hline 6 & 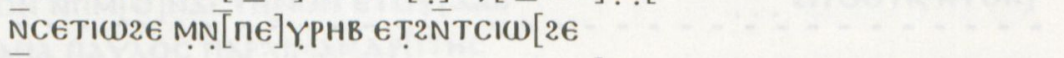 \\
\hline 7 & $\bar{N}$ CETIC2E TINOY OYN TN2OMOAOTI. . [ \\
\hline 8 & 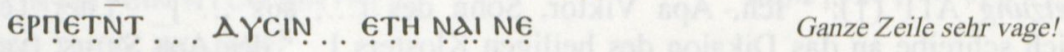 \\
\hline 9 & $\sigma \mu[10 v] \quad \sigma \mu \mu 10 v \quad \sigma \mu 10 v$ \\
\hline & +++10 \\
\hline & 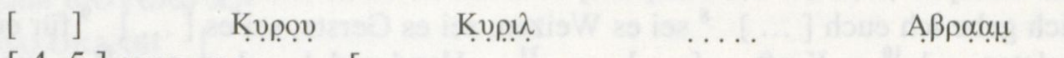 \\
\hline 10 & [4-5 ] X̣̣€ OYN ... . [ [ \\
\hline 11 & 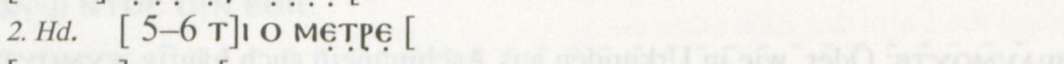 \\
\hline 12 & {$[\quad] \ldots[$} \\
\hline
\end{tabular}


Übersetzung A12 ( $\uparrow):{ }^{1}$ Wir, die Ackerbauern des Apa D ... [.., aus ... ${ }^{2}{ }^{2}$ und Apa ... und ... Abrah[am .... ${ }^{3}$ wir schreiben an das Dikaion des heiligen Klosters [ ... ] ${ }^{4}$... alle ... gemäß [... $]^{5}$ Ackeraruren in ... [ . . ] ${ }^{6}{ }^{6}$ Ackeraruren und ihren Anteil, der in der Ackeraru[re ... $]{ }^{7}$ Ackeraruren. Jetzt nun bekennen wir, ... [ ... ${ }^{8}$ indem euer ..., nämlich [ ... ${ }^{9}$ Handzeichen des [ ... ] +++, Handzeichen des Kyros +++, Handzeichen des Kyril(los) +++, Handzeichen des [ .. ], Handzeichen des Abraam +++ ${ }^{10}[\ldots] \ldots[\ldots]{ }^{11}[\ldots$ ic $]$ h bin Zeuge $[\ldots]{ }^{12}[\ldots] \ldots[\ldots]$

A12 $(\rightarrow)$ : Breitstrichige Schrift in schwarzer Tinte, rechter Rand komplett, oben Text wohl nahezu komplett, links und unten abgebrochen. Teil einer Bürgschaft betreffenden Rechtsurkunde.

\begin{tabular}{|c|c|}
\hline$x+1$ & P]MANATINOOY ПГАגIГPAфOC \\
\hline 2 & 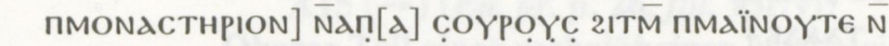 \\
\hline 3 & 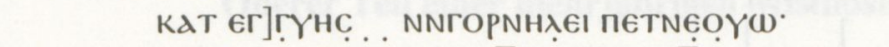 \\
\hline 4 & 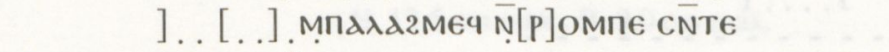 \\
\hline 5 & ]. TחENAITHK,$. \Delta I K=$ \\
\hline 6 & ]. TOYCId' MTMONACTHPION \\
\hline 7 & ] KEAddY EMd NKKdTHGEI \\
\hline 8 & ]TNEGN̄T9 $\triangle \in$ חECMOŌNE ETOY \\
\hline 9 & $(\omega) \mathrm{M}] \omega) \in$ EITE KBd EITE $2 \omega B$ NIM \\
\hline 10 & ]. OC па) $\bar{N} \Pi \Delta B O O Y \bar{N}$ \\
\hline 11 & EIT]€ $(1) \bar{M}(1) € \in$ ЄITE KBd \\
\hline 12 & 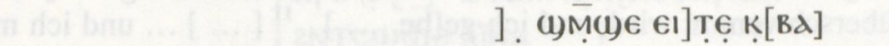 \\
\hline
\end{tabular}

Übersetzung A12 $(\rightarrow):{ }^{1}$ [Ich, ..., Sohn des ..., Ein]wohner von Antinou, der Kalligraph, ${ }^{2}$ [... ich schreibe an das Kloster] des Apa Surus, (vertreten) durch den gottliebenden ${ }^{3}$ [ . . zur Bürg]schaft ... des Kornelios. Das, was ihr verkündet(?), ${ }^{4}[\ldots]$ meines Lahmef, zwei Jahre ${ }^{5}\left[\right.$... ] des fünfzehnten(?) Indiktions-Jahres(?) ${ }^{6}[$. . ] d das Landgut

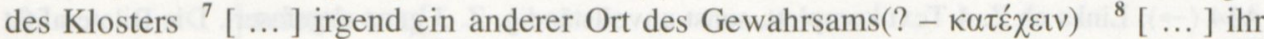
ihn aber finden werdet ....... ${ }^{9}\left[\ldots\right.$ D]ienst oder Zwangsarbeit oder irgendeine Sache ${ }^{10}$ [ ... ]...os, Sohn des Pabou ${ }^{11}$ [ . . ode]r Dienst oder Zwangsarbeit oder irgendeine Sache

${ }^{12}[\ldots]$ Dienst oder Zwangsarbeit

1 Antinou: vgl. Duttenhöfer \& Worp 1996 und vgl. 29.

$4 \mathrm{Zu}$ 入д2м€ч vgl. Crum $C D$ 150a, s.v.: „,meaning unknown, ? relates to pecuniary matters (hire,wage)“.

5 ed.pr. schlägt die Deutung nENTEKגIAEKATHC INAIKTIONOC vor.

7 кдтнбєı: Oder lies хатнбєı?

\section{A13 ( $\uparrow$ (Ed. pr. p. 25-26, fol. 13v): Anfang einer Misthosis (?)}

H. 14,0 cm max.; B. 12,5 cm max.

A13 $(\uparrow): 12$ Zeilen einer Misthosis(?)-Urkunde, oben und wohl auch rechts komplett (von den ersten drei Zeilen jedoch nur Schluss erhalten); unten und links abgebrochen. Teile der Klauseln über das Pachtobjekt und über die Pächterarbeiten. 


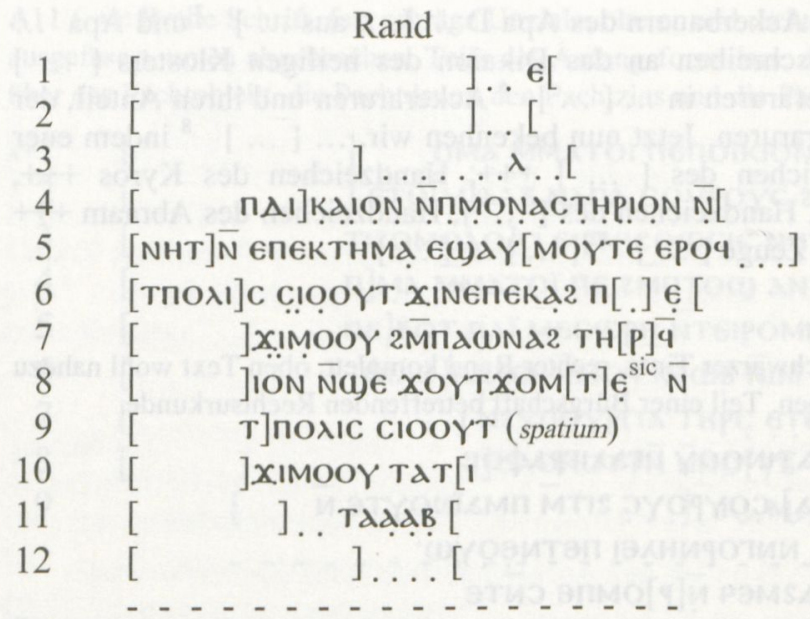

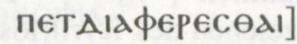

A13 $(\rightarrow)$ : Um $180^{\circ}$ gedreht. Schluss einer griechischen Misthosis-Urkunde, vgl. Duttenhöfer \& Worp 1996, 104-105.

Übersetzung A13 $(\uparrow):{ }^{1}[\ldots] \ldots[\ldots]{ }^{2}[\ldots] \ldots[\ldots]{ }^{3}[\ldots] \ldots[\ldots]{ }^{4}[$ ich schreibe an das Di]kaion des Klosters des [ . . . das, was euch gehört] ${ }^{\mathbf{5}}$ von dem Landgut, das man [nennt: ... ${ }^{6}$ [der Stadt] Siût, von dem Land ... [ ... ] ${ }^{7}[\ldots]$ überschwemmt wird, während meines ganzen Lebens ${ }^{8}[\ldots]$ einhundertdreiundzwanzig ${ }^{9}[\ldots$ der] Stadt Siût. ${ }^{10}[\ldots]$ überschwemmt wird, und ich ge [be ... ${ }^{11}[\ldots] \ldots$ und ich mache ihn $[\ldots]^{12}[\ldots] \ldots[\ldots]$

\section{A14 $(\rightarrow)($ Ed. pr. p. 26, fol. 14r): Aus einer Emphyteusis-Urkunde(?)}

H. 12 max.; B. 12,5 cm max.

A14 ( $\rightarrow$ ): Links ab Z. 4 Text komplett, sonst unvollständig. Z. 3 ganz abgefasert. Die Bilateralität der Ausfertigung deutet auf eine Erbpacht-Urkunde hin (s.u., Anhang).

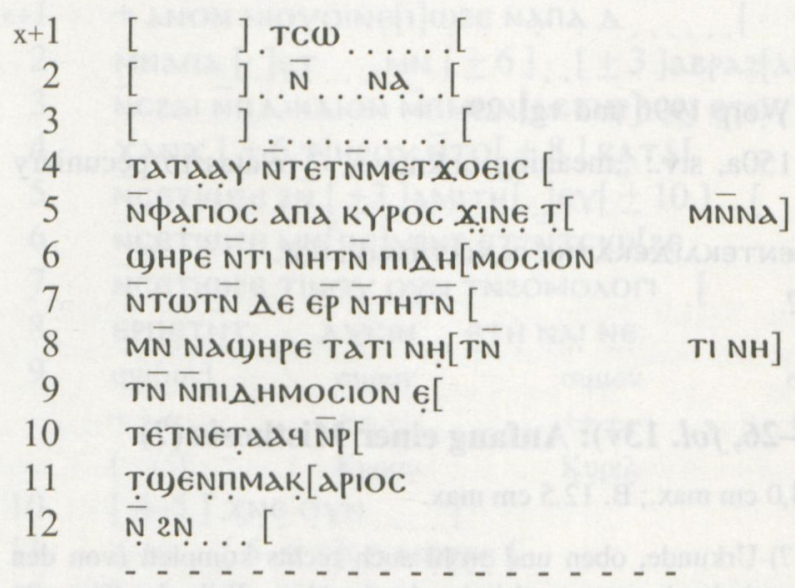


A14 ( $\uparrow):$ Vgl. Duttenhöfer \& Worp 1996, 105.

Übersetzung A14 $(\rightarrow):{ }^{1}[\ldots] \ldots[\ldots]{ }^{2}[\ldots] \ldots[\ldots]{ }^{3}[\ldots] \ldots[\ldots]{ }^{4}$ und ich gebe sie Eurer Herrschaft $[\ldots]{ }^{5}$ des Heiligen Apa Kyros, ab der $[\ldots, \ldots$ mit meinen] ${ }^{6}$ Kindern, euch zu geben diese Gr[undsteuer $\left.(\delta \eta \mu o ́ \sigma t o v) . ..\right]{ }^{7} \mathrm{Ihr}$ aber, zu machen eure [ ... ${ }^{8}$ mit meinen Kindern, dass ich euch gebe [ ... euch zu geben] ${ }^{9}$ diese Grundsteuer $\left(\delta \eta \mu \sigma^{\sigma} \iota_{0}\right)[\ldots]{ }^{10}$ ihr werdet ihn machen zum $\left.[\ldots]\right]^{11}$ die Tochter des sel[igen ... ${ }^{12} \ldots[\ldots]$

11 Lesung na)€ ,der Sohn‘ ebenfalls möglich.

\section{$\mathrm{A15}(\rightarrow)(E d . p r$. p. 26, fol. 15r/v): Oberer Teil einer mehrjährigen Misthosis}

H. 13,5 cm max.; B. 9,0 cm max.

A15 $(\rightarrow)$ : Evtl. oben fast komplett, sonst alle Seiten unvollständig. Z. 1 abgefasert. Große, klare, dünnstrichige Schrift, kaum Ligaturen. Teile von Klauseln über das Pachtobjekt, die Pachtdauer, die Pächterarbeiten und den Pachtzins.

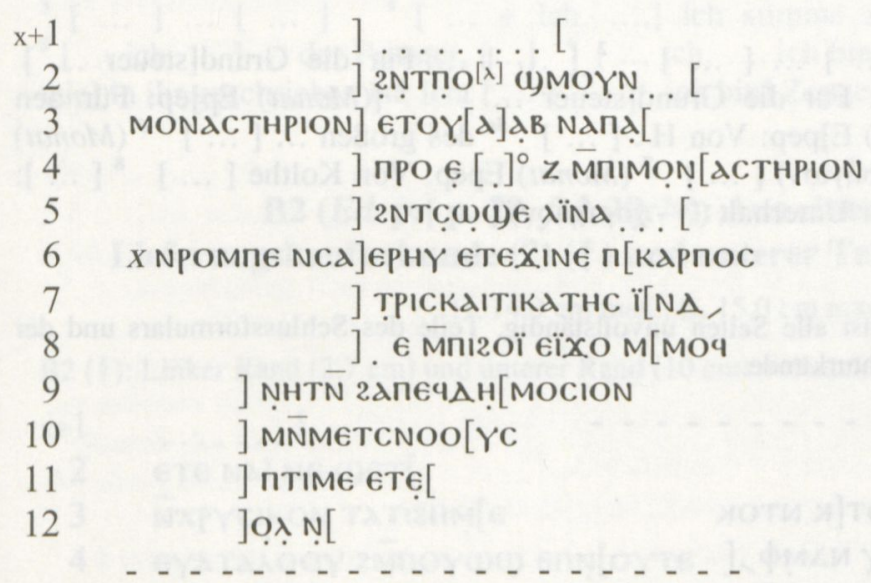

A15 ( $\uparrow$ ) Oben 1 Zeile, wohl Docket; Mitte links weitere Spuren.

1 OYN [..] $] \operatorname{enI} \phi[\ldots] \mathrm{HIN}$

Übersetzung A15 $(\rightarrow / \uparrow):{ }^{1}[\ldots] \ldots[\ldots] \quad{ }^{2}[\ldots]$ in der Stadt Schmoun ... $[\ldots]^{3}$ ich schreibe an das] heilige $[$ Kloster $]$ des Apa $[\ldots]^{4}[\ldots] \ldots$ dieses Klos $[$ ters $\ldots]^{5}[\ldots]$ im Feld ... [ ... ${ }^{6}{ }^{6}[\ldots x$ Jahre hinter $]$ einander, das heißt ab der [Frucht des $x$. Ind.-Jahres... ${ }^{7}[\ldots]$ dreizehnten Ind.-Jahres $[\ldots]{ }^{8}[\ldots]$ dieses (künstlich bewässerten) Feldes, und ich besäe $\mathrm{e}\left[\mathrm{s} \ldots{ } . . .{ }^{9}[\ldots\right.$ und ich gebe $]$ euch für seine Grund[steuer ... $]^{10}[\ldots]$ und zwölf $[\ldots]{ }^{11}[\ldots]$ das Dorf, welches $[\ldots]{ }^{12}[\ldots] \ldots$ [ ... ] Verso: ... (Monat) Epep ... 


\section{A16 (Ed. pr. p. 27, fol. 16r/v): Liste über finanzielle(?) Transaktionen in den Monaten Paone und Epep $(\rightarrow)$ und Ende einer Misthosis $(\uparrow)$}

H. 13,5 cm max.; B. 10,2 cm max.

A16 ( $\uparrow)$ : Links und unten komplett, rechts und oben abgebrochen.

\begin{tabular}{|c|c|c|}
\hline$x+1$ & & ]$N .[$ \\
\hline 2 & ] . & $2 \Delta \Pi \triangle H M[O C I O N$ \\
\hline 3 & ח]גִYNI r & $2 \triangle \Pi \triangle H M O C I O N$ \\
\hline 4 & $\epsilon \Pi] \epsilon ı \phi[1]$ & 2dTTIM $[\mathrm{H}$ \\
\hline 5 & $\epsilon] n \epsilon ı і ̈$ & $21 T \bar{N} 2 .[$ \\
\hline $5 a$ & \multicolumn{2}{|c|}{ NกNOG NA. } \\
\hline 6 & $\epsilon n \in ı \phi 1$ & $2 d X Y P G G \bar{M}$ [ \\
\hline 7 & EnEIфI & $2 І T \bar{N}$ KO \\
\hline 8 & {$\left[\begin{array}{ll}1 & ]\end{array}\right]$} & $2 \lambda T[.] \pi \lambda{ }^{2}[$ \\
\hline 9 & $\begin{array}{l}\text { ] } \\
(S p\end{array}$ & $2 \alpha$ XYPEGM [ \\
\hline
\end{tabular}

Übersetzung A16 (I): ${ }^{1}[\ldots] \ldots[\ldots]{ }^{2}[\ldots]$...: Für die Grund[steuer ... ] ${ }^{3}$ [(Monat) P]aone (Tag) 3: Für die Grund[steuer ... $]{ }^{4}[($ Monat $)$ Ep $]$ ep: Für den Kaufpreis $[\ldots]{ }^{5}[(\text { Monat }) \text { E]pep: Von H . [ ... }]^{5 a}$ des großen ... [ ... $]{ }^{6}{ }^{6}$ Monat) Epep: Für Unterhalt $\left(?-\chi 0 \rho \eta ́ \gamma\right.$ iov) $[\ldots]{ }^{7}$ (Monat) Epep: Von Kolthe $[\ldots]{ }^{8}[\ldots]$ : Für die $\ldots[\ldots]{ }^{9}[\ldots]$ : Für Unterhalt $(?-\chi o p \eta ́ \gamma ı v)[\ldots]$

A16 $(\rightarrow)$ : Unten komplett, sonst alle Seiten unvollständig. Teile des Schlussformulars und der Zeugenunterschriften einer Pachturkunde.

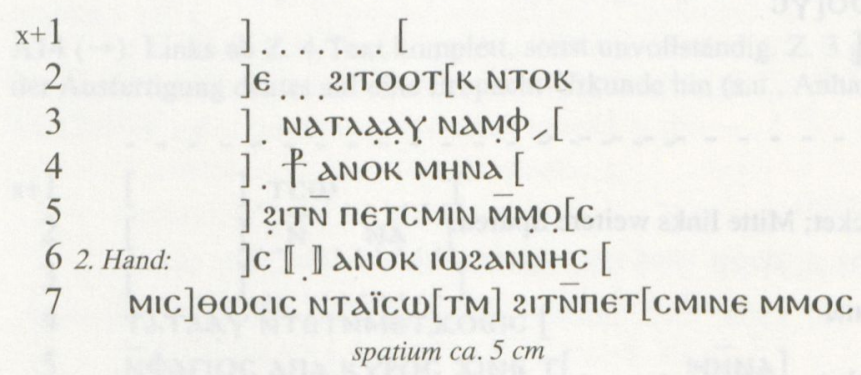




\section{A17 $(\rightarrow)($ Ed. pr. p. 27, fol. 17r): Ende einer Misthosis}

H. $10,5 \mathrm{~cm}$ max.; B. $10,5 \mathrm{~cm}$ max.

A17 $(\rightarrow)$ : Zwei aneinanderpassende Fragmente. Text unten komplett, sonst alle Seiten abgebrochen. Teile der Zustimmung des Pächters und der Zeugenunterschriften einer Pachturkunde.

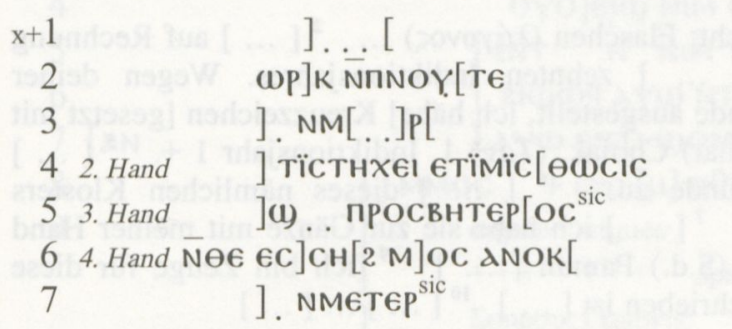

A17 $(\uparrow):$ Schriftspuren

Übersetzung A17 $(\rightarrow):{ }^{1}[\ldots] \ldots[\ldots] \quad{ }^{2}[\ldots$ ich schwö]re bei Gott $[\ldots]$ ${ }^{3}[\ldots] \ldots[\ldots]{ }^{4}[\ldots+$ Ich, ..., $]$ ich stimme zu zu dieser Mis[thosis ...] ${ }^{5}[\ldots$ ich, ... $] \ldots$ der Priester, $[\ldots]{ }^{6}[\ldots$ ich, ..., ich bin Zeuge für diese Misthosis so, wie $]$ in ihr geschrieben ist. Ich, $[\ldots]^{7}[\ldots$ ich bin] Zeuge $[\ldots]$

\section{B2 (Ed. pr. p. 29, fol. 20r/v): Aus einer Pacht- oder}

\section{Lieferungskaufurkunde $(?)(\uparrow)$ und unterer Teil einer Asphaleia $(\rightarrow)$}

H. 14,0 cm max.; B. 15,0 cm max.

B2 ( $\uparrow)$ : Linker Rand $(2,7 \mathrm{~cm})$ und unterer Rand $(10 \mathrm{~cm})$ vollständig, oben und rechts abgebrochen

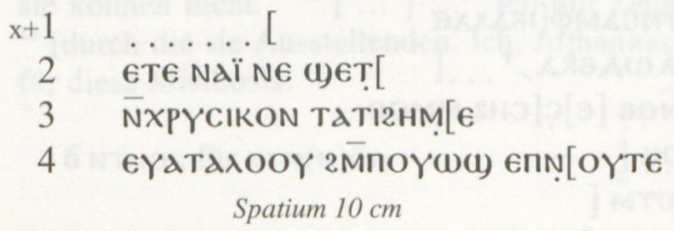

Übersetzung B2 $(\uparrow):{ }^{1} \ldots[\ldots]{ }^{2}$ nämlich die folgenden: ... [ .. ] ${ }^{3}$ als Pachtzins in

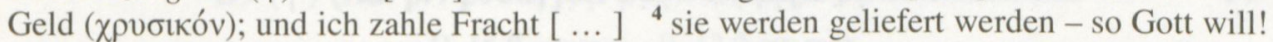
$-,[\ldots]$

B2 $(\rightarrow)$ : Rechter und unterer Rand komplett; links und oben abgebrochen. Kursive mit Ligaturen;

2. Hand sehr unbeholfen.

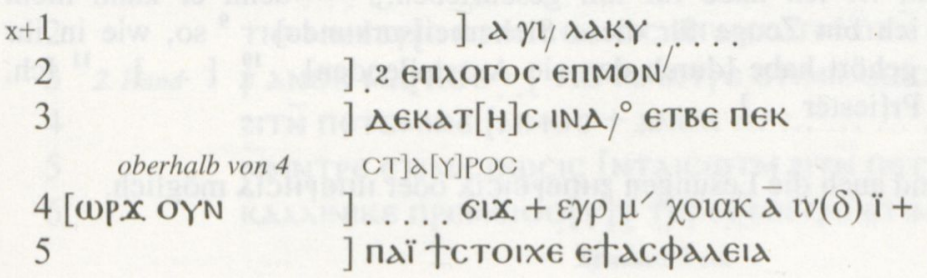




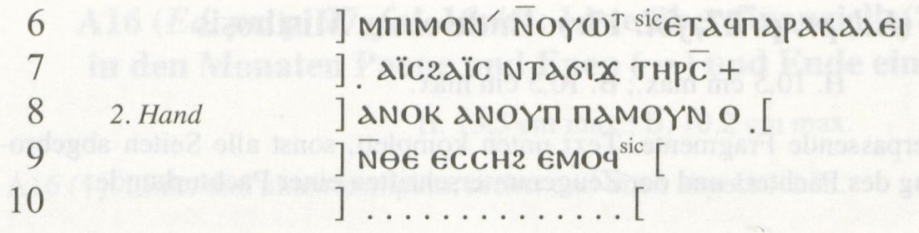

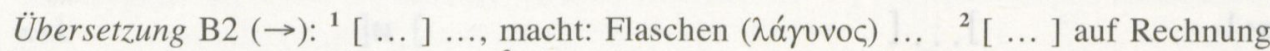
des Klosters ( ${ }^{4}$ [Sicherheit nun habe ich diese Urkunde ausgestellt, ich habe] Kreuzzeichen [gesetzt mit meiner] Hand. Geschrieben (im) M(onat) Choiak, (Tag) 1, Indiktionsjahr $1+.{ }^{5}[\ldots]$ ... ich stimme dieser Sicherheitsurkunde zu. ${ }^{6}$ [ ... ] dieses nämlichen Klosters

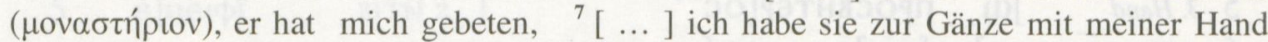
geschrieben.$+{ }^{8}[\ldots]$ Ich, Anup (S.d.) Pamun [ ... ] ${ }^{9}$ [ich bin Zeuge für diese Sicherheitsurkunde $]$ so, wie in ihr geschrieben ist $[\ldots]{ }^{10}[\ldots] \ldots[\ldots]$

\section{B7 (Ed. pr. p. 31-32, fol. 25r/v): Ende einer Asphaleia( $\rightarrow$ ) und Ende einer Misthosis $(\uparrow)$}

H. 14,5 max.; B. 15,5 cm max.

B7 $(\rightarrow)$ : Linker und unterer Rand komplett (unten ausgefasert); rechter Rand und oberer Teil abgebrochen. Schlussformular und Zeugenunterschriften.

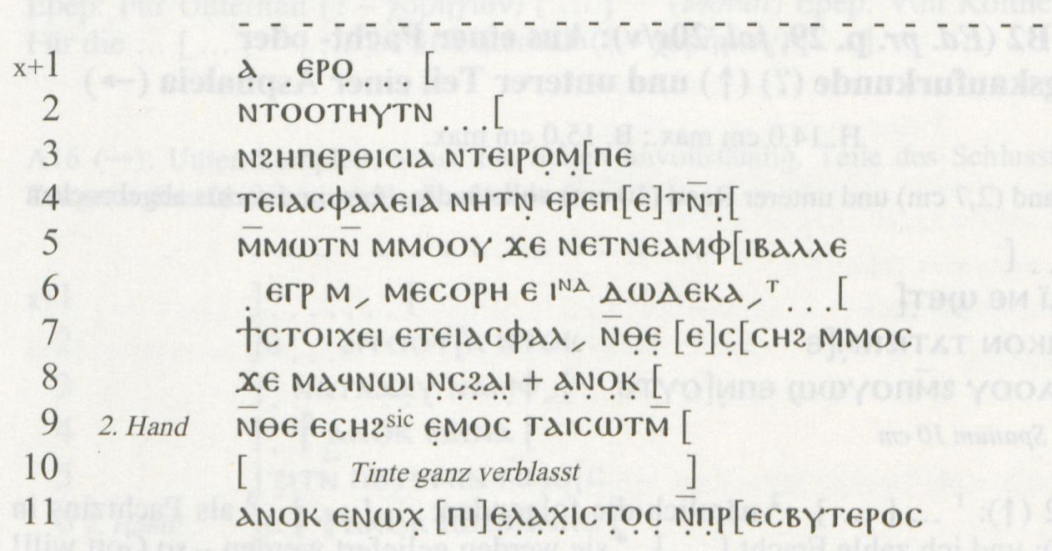

Übersetzung B7 $(\rightarrow):{ }^{1} \ldots[\ldots]{ }^{2}$ durch euch ... [ . . ] ${ }^{3}$ als Aufschub (?- vi $\left.\pi \varepsilon \rho \theta \varepsilon \sigma i ́ \alpha\right)$ dieses Jah[res ... ${ }^{4}$ diese Sicherheitsurkunde für euch, indem euer $[\ldots]^{5}$ euch wegen ihnen; damit ihr nicht zwei[felt ... ] ${ }^{6}$ Geschrieben (im) M(onat) Mesore, (Tag) 5, des zwölften Indiktions-Jahres. ... [ ... ] ${ }^{7}$ Ich stimme dieser Sicherheitsurkunde zu, wie in ihr geschrieben ist. [Ich, ... ich habe für ihn geschrieben,] ${ }^{8}$ denn er kann nicht schreiben. + Ich, [ . ., ich bin Zeuge für diese Sicherheitsurkunde] ${ }^{9}$ so, wie in ihr geschrieben ist, die ich gehört habe [durch den sie Ausstellenden] ${ }^{10}[\ldots]{ }^{11}$ Ich, Enoch, [jener] geringste Pr[iester ... ]

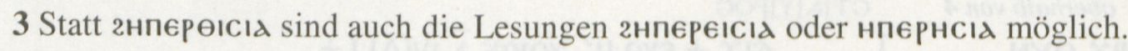


B7 $(\uparrow)$ : Rechter und unterer Rand komplett; linker Rand und oberer Teil abgebrochen. Schlussformular, Zustimmung der Mieter/Pächter und Zeugenunterschriften.

$\mathrm{x}+1$
2
3
4
5
6
7
8
9

$$
\begin{aligned}
& \text { ]E } \bar{N} K \in P \Delta[T] N \\
& \text { ]. } M A N M \bar{N} T \bar{N} \\
& \text { ]. . G KATA POMnE } \\
& \text { oYo] Ẹ़̆(W NIM ETNEOYWW } \\
& \text { ] } 2 \bar{N} T \text {. . .N. NIM TIMIC } \Theta \omega C I C
\end{aligned}
$$

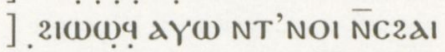

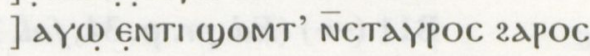

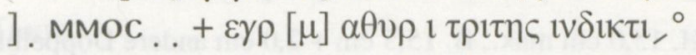

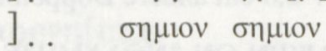

$$
\begin{aligned}
& \text { ] } \quad+++++ \text { Spatium } 8 \mathrm{~cm}
\end{aligned}
$$

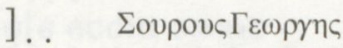

10

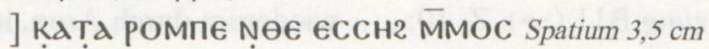

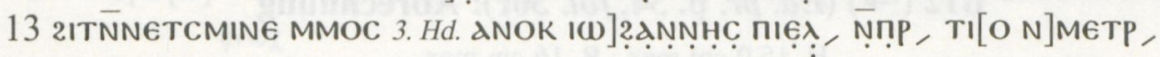
ETIMIC $\theta \omega$

\section{Spatium $2 \mathrm{~cm}$}

Übersetzung B7 $(\uparrow):{ }^{1}[\ldots]$ Karat ${ }^{2}[\ldots] \ldots$ und unsere ${ }^{3}[\ldots] \ldots$ pro Jahr ${ }^{4}[\ldots]$ zu jeder Zeit, da Ihr wollt, ${ }^{5}[\ldots]$ in jeder ... Diese Misthosis ${ }^{6}[\ldots]$ auf ihm, und wir können nicht schreiben ${ }^{7}[\ldots]$ und wir setzen drei Kreuze darunter. ${ }^{8}[\ldots]$ ... + Geschrieben (im) M(onat) Hathor, (Tag) 10, des dritten Indiktions-Jahres. ${ }^{9}$ [ ... ] ... Handzeichen des Surus: +++ , Handzeichen des Georgios:.$+++{ }^{10}[\ldots]$ pro Jahr, wie in ihr geschrieben ist. ${ }^{11}[$... sie haben mich ge]beten, ich habe für sie geschrieben, denn sie können nicht. ${ }^{12}[\ldots] \ldots$, ich bin Zeuge für diese Misthosis, die ich gehört habe ${ }^{13}$ [durch die sie Ausstellenden. Ich, Jo]hannes, jener geringste Pr(iester), ich [bin] Zeuge für diese Misthosis.

6 NTNOI: für NTN(N)OI.

\section{B9 ( $\uparrow)($ Ed. pr. p. 32, fol. 27r): Schluss einer Misthosis}

H. 15,0 cm max.; B. 13,0 cm max.

B9 ( $\uparrow)$ : Linker und unterer Rand komplett; rechter Rand und oberer Teil abgebrochen. Zeugenunterschriften.

$\begin{array}{rr}x+1 & \\ 2 & \\ 3 & 2 . \text { Hand } \\ 4 & \\ 5 & \\ 6 & \end{array}$

$$
+ \text { dṆỌK }[
$$

TIO MMETP[E ETIMIC

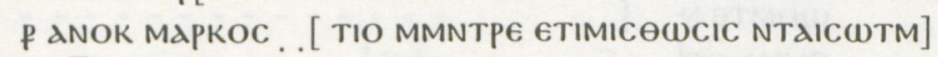
IITN $\bar{N}$ пTCMINE $[$ MMOC + ANOK $\ldots \ldots \ldots \ldots \ldots \ldots \ldots \ldots \ldots$ TIO $]$ MMNTPE ETIMIC

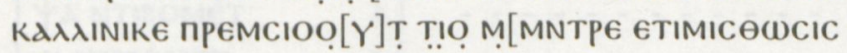


B9 $(\rightarrow)$ : Vgl. Duttenhöfer \& Worp 1996, 105.

Übersetzung B9 ( $\uparrow):{ }^{1}+$ Ich, [ . . ] ${ }^{2}$ ich bin Zeug[e für diese Misthosis, die ich gehört habe durch den sie Ausstellenden.] ${ }^{3}+$ Ich, Markos, ... [bin Zeuge für diese Misthosis, die ich gehört habe] ${ }^{4}$ durch den sie Ausstellenden. [+ Ich, ... ich bin ] ${ }^{5}$ Zeuge für diese Misthosis, [die ich gehört habe durch den sie Ausstellenden. + Ich, ${ }^{6}$ Kallinikos, Einwohner von Siût, ich bin Zeuge für diese Misthosis.

\section{$\mathrm{B} 11(\rightarrow)($ Ed. pr. p. 34, fol. 29v)}

H. 15,0 cm max.; B. 15,5 cm +2,0 cm andere Doppelblatthälfte
$10(=e d \cdot p r .9)$
]KH $21 T \bar{N}$ APWN ON $2 \bar{N} \theta d \lambda M H 2 € \bar{N}$

Übersetzung B11 $(\rightarrow)$, Z. 10: „... wiederum durch Aron, in dem Schiff des ...“

\section{B12 $(\rightarrow)(E d . p r$. p. 34, fol. 30r): Abrechnung}

H. 15,0 cm max.; B. $16 \mathrm{~cm} \max$.

B12 $(\rightarrow)$ : Rechter und oberer(?) Rand komplett; linker Rand und unterer Teil abgebrochen.

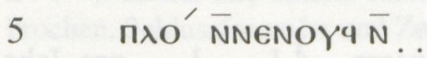

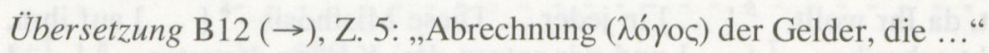

\section{B13 (Ed.pr. p. 34-35, fol. 31r/v): Geräteliste $(\rightarrow)$ und Schluss einer Misthosis mit fünf Jahren Laufzeit $(\uparrow)$}

H. 14,5 cm max.; B. 15,5 cm max. (davon evtl. 2-3 cm andere Blatthälfte: links 5,5 cm Rand) B13 $(\rightarrow)$ :

\begin{tabular}{|c|c|}
\hline$x+1$ & OYN ...N OYP.[ \\
\hline 2 & 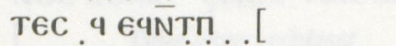 \\
\hline 3 & OYC YYPIN NBEPE. [ \\
\hline 4 & Ф)ОМТє NTт4є = \\
\hline 5 & OYNOG NTAOOGE [ \\
\hline 6 & OYそHAON NTNTA [ \\
\hline 7 & OYNOE NMEPE EC̣OYOX. \\
\hline 8 & MANAKOT CNAY NBIPE [ \\
\hline 9 & OYKANKH入 $\bar{N}(1) € N[$ \\
\hline 10 & OYQOPION E46[ \\
\hline 11 & ФOMTE $\bar{N} \ldots[$ \\
\hline 12 & OYMA N $G[$ \\
\hline 13 & $\begin{array}{l}\text { OY.. [ } \\
-\ldots-\ldots-\ldots\end{array}$ \\
\hline
\end{tabular}


Übersetzung B13 $(\rightarrow):{ }^{1} \ldots[\ldots]^{2} \ldots[\ldots]^{3}$ Ein neues ... [ . . ] ${ }^{4}$ Drei Kästen $[\ldots]$

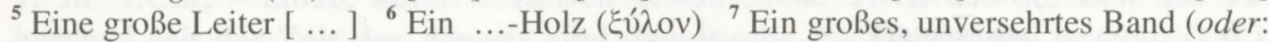

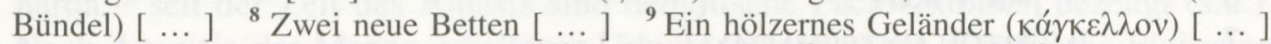
${ }^{10}$ Eine Trage (

B13 ( $\uparrow):$ Um $180^{\circ}$ gedreht; linker Rand komplett; unterer Rand komplett, doch ausgefasert; rechter Rand und oberer Teil abgebrochen und ausgefasert. Teile der Klausel über den Kündigungsverzicht, des Schlussformulars, der Zustimmung der Pächterseite und der Zeugenunterschriften.

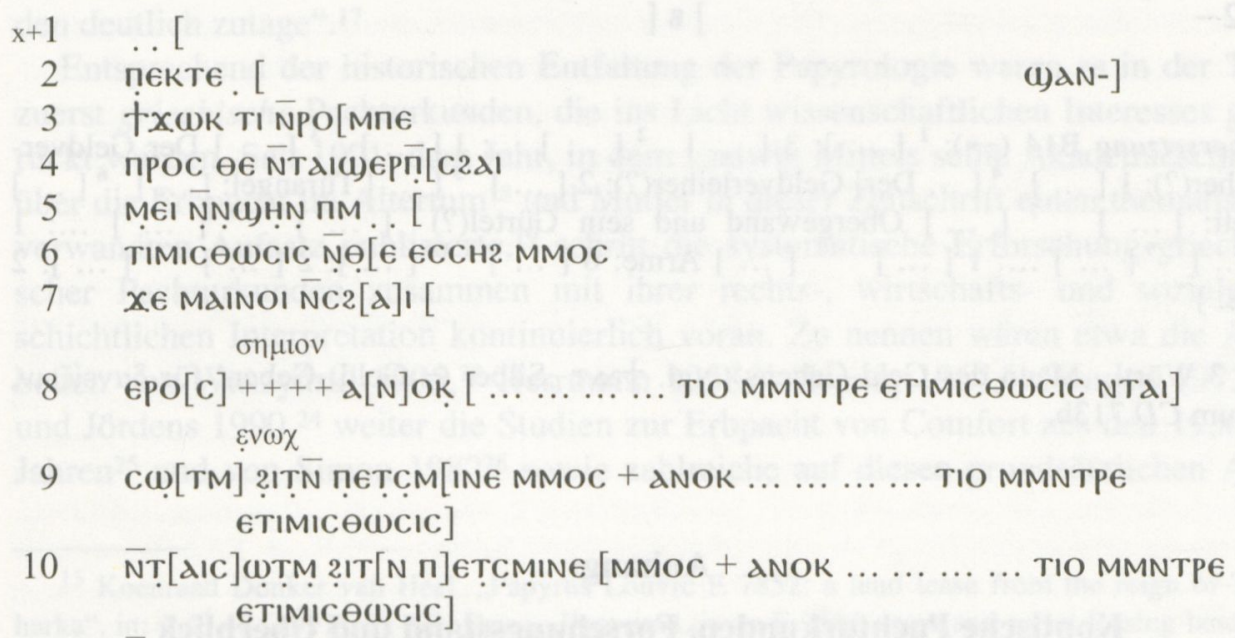

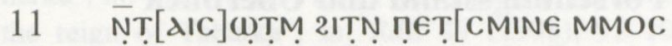

12 (evtl. nur verrutschte $\rightarrow$ Fasern von $Z$. 11)

Übersetzung B13 $(\uparrow):{ }^{1} \ldots[\ldots]^{2} \ldots\left[\ldots\right.$, bis ${ }^{2}{ }^{3}$ ich vollende $<$ diese $>$ fünf Jah $[\mathrm{re} \ldots$ ] ${ }^{4}$ so, wie ich bereits [geschrieben habe ... ] ${ }^{5} \ldots$ die Bäume ... [ ... ] ${ }^{6}$ diese Misthosis, wie [in ihr geschrieben ist ... ] ${ }^{7}$ denn ich kann nicht schrei[ben ... Ich, Enoch, stimme zu] ${ }^{8}$ zu ihr! Handzeichen des Enoch +++. + Ich, [ ..., ich bin Zeuge für diese Misthosis, die ich] ${ }^{9}$ gehört habe durch den sie Ausstellen[den. + Ich, ... ich bin Zeuge für diese Misthosis,] ${ }^{10}$ die ich gehört habe durch den [sie Ausstellenden. + Ich, ... ich bin Zeuge für diese Misthosis,] ${ }^{11}$ die ich gehört habe durch den [sie Ausstellenden ... ] 12 ...

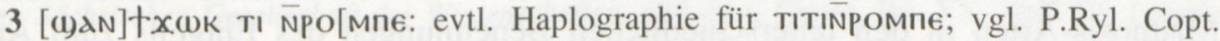

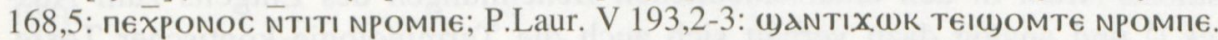

\section{B14 $(\rightarrow)$ (Ed. pr. p. 35, fol. 32v): Liste}

H. 13,0 cm max.; B. 15,5 cm max.

B14 ( $\rightarrow$ ): Reste einer Rechnung, wohl an allen vier Seiten unvollständig.

\begin{tabular}{|c|c|}
\hline$x+1$ & \\
\hline 2 & ZIME กג2(1)€ \\
\hline 3 & $\psi_{\lambda} \bar{N} T I 2 O M E T$ \\
\hline 4 & N NTTI2OMET \\
\hline
\end{tabular}




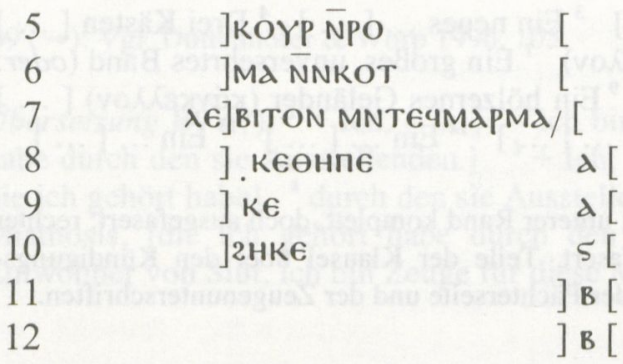

Übersetzung B14 $(\rightarrow):{ }^{1}[\ldots]: 3[\ldots]{ }^{2}[\ldots] \ldots: 1[\ldots]{ }^{3}[\ldots]$ Der Geldverleiher(?): $1[\ldots]{ }^{4}\left[\ldots\right.$ Der] Geldverleiher(?): $2[\ldots]{ }^{5}[\ldots]$ Türangel: $[\ldots]{ }^{6}[\ldots]$ Bett: $[\ldots]{ }^{7}[\ldots]$ Obergewand und sein Gürtel(?) $[\ldots]^{8}[\ldots] \ldots$. 1 $[\ldots]{ }^{9}[\ldots] \ldots: 1[\ldots]{ }^{10}[\ldots]$ Arme: $6[\ldots]{ }^{11}[\ldots]: 2[\ldots]{ }^{12}[\ldots]: 2$ $[\ldots]$

3 Wörtl. ,Mann des Geld-Gebens'; vgl. †2גт ,Silber (= Geld)-Geben` für $\delta \alpha v \varepsilon i ́ \zeta \varepsilon ı v$, Crum $C D 713$ b.

\section{Anhang:}

\section{Koptische Pachturkunden. Forschungsstand und Überblick}

Pacht, die zeitlich befristete Übergabe einer Sache gegen Entgelt zum Zweck des usus fructus, jouissance, usufruit, Nutzbrauch, Nießbrauch, Fruchtgenuß, um in der Terminologie des römischen Rechts zu sprechen, ist eine in der papyrologischen Überlieferung reich bezeugte ökonomische Strategie. In den griechischen Papyri aus Äypten begegnet uns Pacht als eine Art der Misthosis, die daneben auch Miete, d.h. die befristete, entgeltliche Überlassung einer Sache zum einfachen Gebrauch ohne Fruchtgenuß, und Werkverträge einschloss und in dieser Hinsicht der römischrechtlichen locatio conductio in ihren Typen der locatio conductio rei und operis entsprach. ${ }^{12}$ Wurde zwischen Miete und Pacht demnach nicht kategorisch unterschieden, so kam doch der Unterschied dieser beiden Misthosis-Arten in den unterschiedlichen Benennungen des Entgelts zum Ausdruck, ${ }^{13}$ und analog dazu verhält sich auch die Terminologie koptischer Texte, worauf zurückzukommen sein wird. ${ }^{14}$

Die am häufigsten zur Nutzung übereignete Sache war fruchtbarer Boden. Bodenpacht läßt sich in Ägypten wenigstens bis ins Neue Reich zurückverfolgen, wo Tempeldomänen in einem der Pacht ähnlichen Modus kultiviert und bewirtschaftet wurden. Die ältesten schriftlichen Pachtvereinbarungen stammen aus dem

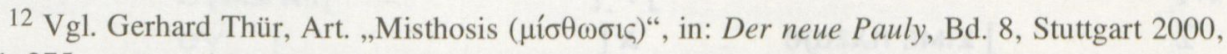
271-275.

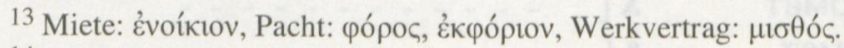

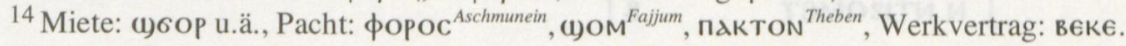


7. Jh. v.Chr, - kurze, kursivhieratisch geschriebene Texte aus der Zeit des Taharqa; ${ }^{15}$ seit der Zeit des Amasis sind demotische Pachturkunden bezeugt (s.u.). Nach Ausweis der Menge erhaltener Urkunden scheint die schriftliche Fixierung von Bodenpacht-Vereinbarungen aber erst seit der Ptolemäerzeit häufiger oder gar häufig geworden zu sein. ${ }^{16} 1958$, als ungefähr 450 griechische Pachturkunden aus ptolemäischer, römischer und byzantinischer Zeit bekannt waren, schrieb Johannes Herrmann: „Schon zahlenmäßig tritt die Bedeutung der Pachtvertragsurkunden deutlich zutage". ${ }^{17}$

Entsprechend der historischen Entfaltung der Papyrologie waren es in der Tat zuerst griechische Pachturkunden, die ins Licht wissenschaftlichen Interesses gerückt wurden. Seit 1901, dem Jahr, in dem Ludwig Mitteis seine Akademieschrift über die Erbpacht im Altertum ${ }^{18}$ und Muller in dieser Zeitschrift einen thematisch verwandten Aufsatz publizierte, ${ }^{19}$ schritt die systematische Erforschung griechischer Pachturkunden zusammen mit ihrer rechts-, wirtschafts- und sozialgeschichtlichen Interpretation kontinuierlich voran. $\mathrm{Zu}$ nennen wären etwa die $\mathrm{Ar}$ beiten von Waszyński $1905,{ }^{20}$ Herrmann $1958,{ }^{21}$ Hennig 1967, ${ }^{22}$ Hengstl $1972{ }^{23}$ und Jördens 1990, ${ }^{24}$ weiter die Studien zur Erbpacht von Comfort aus den 1930er Jahren ${ }^{25}$ und von Simon $1982^{26}$ sowie zahlreiche auf diesen grundsätzlichen Ar-

15 Koenraad Donker van Heel, „Papyrus Louvre E 7852: a land lease from the reign of Taharka“, in: $R d E ́$ 48 (1997), 81-93; Ders., „Papyrus Louvre E 7586 verso and recto: leasing land in the reign of Taharka“, in: RdÉ 49 (1998), 91-105; Ders., „Kushite Abnormal Hieratic Land Leases", in: Christopher J. Eyre (ed.), Proceedings of the Seventh International Congress of Egyptologists, OLA 82, Leuven 1998, 339-343; Ders., „Use of Land in the Kushite and Saite Periods", in: B. Haring, R. de Maaijer (eds.), Landless and Hungry? Access to Land in Early and Traditional Societies. CNWS Publications 67 (1998), 90-102; Ders., „Papyrus Louvre E 7581 recto and verso: two more land leases from the reign of Taharka“, in: RdÉ 50 (1999), 135-147.

${ }^{16}$ Zum Normalfall der unbeurkundeten Pacht vgl. Christopher Eyre, „Peasants and ,modern“ leasing strategies in Ancient Egypt“, in: Journal of the Economic and Social History of the Orient 40 (1997), 367-390.

17 Johannes Herrmann, Studien zur Bodenpacht im Recht der graeco-aegyptischen Papyri. Münchener Beiträge zur Papyrusforschung und antiken Rechtsgeschichte 41, München 1958, 8.

${ }^{18}$ Ludwig Mitteis, „Zur Geschichte der Erbpacht im Alterthum“, Abhandlungen der Königlichen Sächsischen Gesellschaft der Wissenschaften, phil.-hist. Classe, Bd. XX, Abh. IV, Leipzig 1901.

${ }^{19}$ H.C. Muller, „Über die von Kenyon herausgegebene Emphyteusis-Urkunde auf Papyrus aus dem J. 616 n.Chr.“, APF 1 (1901), 437-444.

20 Stefan Waszyński, Die Bodenpacht. Agrargeschichtliche Papyrusstudien, 1: Die Privatpacht. Leipzig, Berlin 1905.

${ }^{21}$ Herrmann, Studien zur Bodenpacht im Recht der graeco-aegyptischen Papyri (s.o., Fn. 17).

22 Dieter Hennig, Untersuchungen zur Bodenpacht im ptolemäisch-römischen Ägypten. Diss. München 1967.

23 Joachim Hengstl, Private Arbeitsverhältnisse freier Personen in den hellenistischen Papyri bis Diokletian. Diss. Bonn 1972.

24 Andrea Jördens. Vertragliche Regelungen von Arbeiten im späten griechischsprachigen Ägypten. P.Heid. V, Heidelberg 1990.

${ }^{25}$ Howard Comfort, „Prolegomena to a Study of late Byzantine Land-Leases“, in: Aegyptus 13

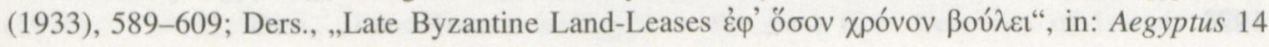


beiten aufbauende Untersuchungen, angefangen mit Schnebels Standardwerk zur Landwirtschaft im hellenistischen Ägypten ${ }^{27}$ über die agrar- und wirtschaftsgeschichtlichen Arbeiten von Rathbone, ${ }^{28}$ Ruffing $^{29}$ oder Wispszycka ${ }^{30}$ bis hin zu Rowlandsons sozialgeschichtlichen Studien, ${ }^{31}$ um nur einige zu nennen. Dabei wurden auch die großen Themen der antiken Wirtschaftsgeschichte wie die Bedeutung der large estates und des Kolonats im spätrömischen und byzantinischen Imperium oder der Streit zwischen Primitivisten und Modernisten aus der Perspektive der graeco-ägyptischen Quellen kommentiert, wie etwa in den Arbeiten von Banaji ${ }^{32}$ und Jördens ${ }^{33}$. Die Auswertung der demotischen Papyri zur Bodenpacht begann erst ein halbes Jahrhundert später mit den Arbeiten von Hughes ${ }^{34}$ und Malinine ${ }^{35}$. Doch in den Untersuchungen von Seidl, ${ }^{36}$ Kaplony-Heckel, ${ }^{37}$

(1934), 80-88; Ders., „Notes on «Requests» and ХЕІРОГРАФА among late Byzantine LandLeases“, in: Aegyptus 14 (1934), 286-292; Ders., „The Crops of an Indiction in late Byzantine Land-Leases“, in: Aegyptus 14 (1934), 429-446: Ders., „Emphyteusis among the Papyri“, in: Aegyptus 17 (1937), 3-24.

26 Dieter Simon, „Das frühbyzantinische Emphyteuserecht“, in: J. Modrzejewski, D. Liebs (eds.), Vorträge zur griechischen und hellenistischen Rechtsgeschichte, Chantilly 1.-4.6.1977, Akten der Gesellschaft für griechische und hellenistische Rechtsgeschichte 3. Köln, Wien 1982, $365-422$.

${ }^{27}$ Manfred Schnebel, Die Landwirtschaft im hellenistischen Ägypten. Münchener Beiträge zur Papyrusforschung und antiken Rechtsgeschichte 7. München 1925.

${ }^{28}$ Dominique Rathbone, Economic rationalism and rural society in third-century A.D. Egypt: The Heroninos archive and the Appianus estate. Cambridge 1991.

${ }^{29}$ Kai Ruffing, Weinbau im römischen Ägypten, St. Katharinen 1999.

${ }^{30}$ Ewa Wipszycka, Les ressources et activités économiques des églises en Égypte du IVe au VIIIe siècle. Pap. Brux 10. Bruxelles 1972.

31 Jane Rolandson, „Crop Rotation and Rent Payment in Oxyrhynchite Land Leases: Social and Economic Interpretations", in: A. Bülow-Jacobsen (ed.), Proceedings of the 20th International Congress of Papyrologists, Copenhagen 23-29 August 1992. Copenhagen 1994, 495-499; Dies., Landowners and Tenants in Roman Egypt: The Social Relations of Agriculture in the Oxyrhynchite Nome. Oxford 1996; Dies., „Agricultural Tenancy and Village Society in Roman Egypt“, in: Alan K. Bowman \& E. Rogan (eds.) Agriculture in Egypt from Pharaonic to Modern Times. Proceedings of the British Academy 96. Oxford 1999, 139-158.

32 Jairus Banaji, „Agrarian History and the Labour Organisation of Byzantine Large Estates“, in: Alan K. Bowman \& E. Rogan (eds.) Agriculture in Egypt from Pharaonic to Modern Times. Proceedings of the British Academy 96. Oxford 1999, 193-216; Ders., Agrarian Change in Late Antiquity: Gold, Labour, and Aristocratic Dominance. Oxford 2001.

33 Andrea Jördens, „Die Agrarverhältnisse im spätantiken Ägypten“, in: Laverna 10 (1999), 114-152.

34 George R. Hughes, Saite Demotic Land Leases, SAOC 28, Chicago/Ill. 1952; Ders. 1973. „Notes on Egyptian Demotic Leases of Property“, in: JNES 32, 152-160.

${ }^{35}$ Michel Malinine, „Trois documents de l'époque d'Amasis relatifs au louage de terres“, $R d E$ 8 (1951), 127-150; Ders., Choix des textes juridiques en hiératique «anormal» et en démotique, $1^{\text {ière }}$ partie, Paris 1953.

${ }^{36}$ Erwin Seidl, Bodennutzung und Bodenpacht nach den demotischen Texten der Ptolemäerzeit. Österreichische Akademie der Wissenschaften, Sitzungsberichte der phil.-hist. Kl., 291. Wien 1973. 
Martin, ${ }^{38}$ Felber, ${ }^{39}$ Manning ${ }^{40}$ und Mrsich $^{41}$ hat dieser Zweig der papyrologischen Forschung seitdem einen nachhaltigen Aufschwung genommen. Anders wieder die Situation in der seit einigen Jahren florierenden arabischen Papyrologie. Bis in die achtziger Jahre des 20. Jhs. hinein konnte Pacht nach arabischen Papyri beinahe ausschließlich anhand von Grohmanns Editionen arabischer Pachturkunden studiert werden. ${ }^{42}$ Diese Situation hat sich in den letzten 20 Jahren beinahe dramatisch verbessert, und diese Entwicklung ist wesentlich mit dem Namen von Gladys Frantz-Murphy verbunden. ${ }^{43}$ In ihrem 2001 erschienenen Band $C P R$ XXI

37 Ursula Kaplony-Heckel, Die demotischen Gebelen-Urkunden der Heidelberger Papyrussammlung. Veröffentlichungen aus der Heidelberger Papyrussammlung NF 4. Heidelberg 1964; Dies., „Die demotischen Gebelên-Papyri der Berliner Papyrussammlung“, in: Forschungen und Berichte 8 (1967), 70-87; Dies., „Theben Ost I: Acker-Schreiber und Familien-Archive nach den demotischen $r$-rh $=w$ Ostraka“, in: ZÄS 120 (1993), 42-71; Dies., ,Thebanische Acker-Amt-Quittungen“, in: Schafik Allam (Hsgr.), Grund und Boden in Altägypten (Rechtliche und sozio-ökonomische Verhältnisse). Akten des internationalen Symposions Tübingen 18.-20. Juni 1990. Untersuchungen zum Rechtsleben im Alten Ägypten 2, Tübingen 1994, 189-197; Dies., „Zur Landwirtschaft in Oberägypten. Demotische Akten und Urkunden aus Gebelein (II. Jh. v.Chr.) und der arabische Leitfaden des Mahzūmì († 1189 n.Chr.) “, in: I. Shirun-Grumach, Jerusalem Studies in Egyptology, ÄAT 40, Wiesbaden 1998, 57-65; Dies., „Theben-Ost II: Zwölf neue $r-r h=w$ Quittungen und fünf Kurz-Quittungen aus dem Acker-Amt“, in: ZÄS 126 (1999), 41-54; Dies., „Theben-Ost III: Die $r-r h=w$-Tempel-Quittungen und ähnliche Texte. Erster Teil: Allgemeiner Teil und Texte Nr. 18-25“, in: ZÄS 128 (2001), 24-40; Dies., "Theben-Ost III: Die $r-r h=w$-Tempel-Quittungen und ähnliche Texte. Zweiter Teil: Neunzehn $r$-r $h=w$-Tempel-Quittungen (Nr. 2644), eine staatliche $r-r h=w$-Quittung $(* \mathrm{Nr}$. 30A) und drei $i n j$-Tempel-Quittungen $(\mathrm{Nr} . * 35 \mathrm{~A}, 4$, 46)“, in: ZÄS 133 (2006), 34-50.

38 Carry J. Martin, ,Marriage and Family Law in Ancient Egypt, II: Marriages, Wills, and Leases of Land. Some Notes on the Formulae of Demotic Contracts", in: M.J. Geller, H. Maehler (eds.), Legal Documents of the Hellenistic World, London 1995, 58-78.

${ }^{39}$ Heinz Felber, Demotische Ackerpachtverträge der Ptolemäerzeit. Untersuchungen zu Aufbau, Entwicklung und inhaltlichen Aspekten einer Gruppe von demotischen Urkunden. Ägyptologische Abhandlungen 58. Wiesbaden 1997; Ders., „Die Daten der demotischen Ackerpachtverträge der Ptolemäerzeit und das landwirtschaftliche Jahr", in: Akten des 21. Internationalen Papyrologenkongresses Berlin 1995, APF Beihefte 3. Stuttgart, Leipzig 1997, 281-289.

40 Joseph G. Manning, „The Land-Tenure Regime in Ptolemaic Upper Egpyt“, in: Alan K. Bowman \& E. Rogan (eds.) Agriculture in Egypt from Pharaonic to Modern Times. Proceedings of the British Academy 96. Oxford 1999, 83-105; Ders., Land and Power in Ptolemaic Egypt. The Structure of Land Tenure. Cambridge 2003.

${ }^{41}$ Tycho Q. Mrsich, Rechtsgeschichtliches zur Ackerverpachtung auf Tempelland nach demotischem Formular. Österreichische Akademie der Wissenschaften, Sitzungsberichte der phil.-hist. Kl. 703 = Veröffentlichungen der Kommission für antike Rechtsgeschichte 10. Wien 2003.

42 Adolf Grohmann, Arabic Papyri in the Egyptian Library (= APEL), Vol. I-VI, Cairo 1934 1962, bes. APEL II von 1937.

43 Gladys Frantz-Murphy, „Land Tenure and Social Transformation in Early Islamic Egypt“, in: T. Khalidi (ed.), Land Tenure and Social transformation in the Middle East, Beirut 1984, 131139; Dies., The Agrarian Administration of Egypt from the Arab conquest to the Ottomans, Le Caire 1986; Dies., „Papyrus agricultural contracts in the Oriental Institute Museum from Third/ Ninth century Egypt", in: Itinéraires d'Orient. Hommages à Claude Cahen, Res Orientalis 6 (1994), 119-131; Dies., „Land-Tenure in Egypt in the First Five Centuries of Islamic Rule 
legte Frantz-Murphy zuletzt eine umfangreiche Edition und Monographie zur Pacht nach arabischen Papyri und die damit verbundenen Fragen vor. ${ }^{44}$

Alle bis hierher genannten Zweige der Papyrologie sind in der Bearbeitung und Auswertung von Pachturkunden weiter gediehen als die koptische Papyrologie. Aus verschiedenen Gründen ist das seit langem in mehr oder weniger geglückten Editionen vorliegende Dossier koptischer Dokumente zur Pacht nie zusammengestellt, geschweige denn auch nur ansatzweise ausgewertet worden. Um nur einige dieser Gründe zu nennen:

1. Mehr als die Hälfte aller koptischen Bodenpachturkunden stammt aus dem Raum von Aschmunein. Anders als die Papyrusurkunden aus dem früharabischen Djême sind koptische Rechtsurkunden dieser Provenienz oft sehr schlecht erhalten, und so ist uns nicht eine einzige koptische Pachturkunde von dort vollständig überliefert. Nur durch die Zusammenstellung des ganzen Dossiers kann das Repertoire an Fomularen, Formeln und Klauseln überblickt und können die für sich genommen oft wenig ergiebigen Fragmente rekonstruiert werden.

2. Die erste Geschäftsklausel aller Misthosis-Urkunden aus Aschmunein, die Miet- oder Pachterklärung, enthält die Worte $6=1-M i c e 0\}$ Nd=K. Im Griechischen bezeichnet bekanntlich der Aktivstamm $\mu \iota \theta 0$ ov̂v die Perspektive des Verpächters oder Vermieters und bedeutet also verpachten bzw. vermieten, während die Perspektive des Mieters bzw. Pächters dem Mediumstamm $\mu$ ro $\theta$ ov̂ $\sigma \theta \alpha$ l eignet. Den beiden in koptische Quellen eingearbeiteten Rechtshistorikern Arthur Schiller und Artur Steinwenter war nicht klar genug, dass der sahidische Dialekt fast ausschließlich mit dem Aktivstamm griechischer Lehnverben operiert und auch Medium-Bedeutungen durch Aktivstämme auszudrücken pflegt. So bedeutet also $\epsilon=\mathrm{MI}-\operatorname{ce}$ OY $\mathrm{N} d=\mathrm{K}$ immer, ich miete/pachte von $\operatorname{dir}^{6}{ }^{45}$ Die Ausführungen zu Miete und Pacht in den klassischen synthetischen Arbeiten zur koptischen juristischen Papyrologie von Schiller ${ }^{46}$ und Steinwenter ${ }^{47}$ fanden dadurch nicht zu wünschenswerter Klarheit, nämlich zu der Erkenntnis, daß alle koptischen Misthosis-Urkunden Pächter-Urkunden sind, also wie ihre griechischen Modelle im subjektiven Homologie-Stil all das nennen, wozu sich der Pächter gegenüber dem Verpächter verpflichtete.

(Seventh-Twelfth centuries AD)“, in: Alan K. Bowman \& E. Rogan (eds.) Agriculture in Egypt from Pharaonic to Modern Times. Proceedings of the British Academy 96. Oxford 1999, 237-266.

${ }^{44}$ Gladys Frantz-Murphy, Arabic Agricultural Leases and Tax Receipts from Egypt, 148-427 A.H./765-1035 A.D., CPR XXI. Wien 2001.

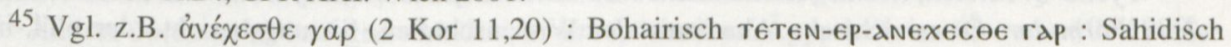

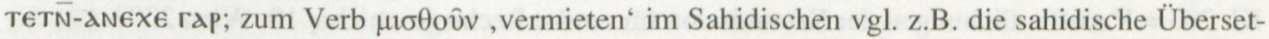

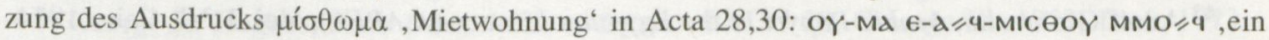
Ort, den er gemietet hatte'.

46 A. Arthur Schiller, „Coptic Law“, in: The Juridical Review, September 1931, 211-240; Ders., „Koptisches Recht. Eine Studie auf Grund der Quellen und Abhandlungen“, in: Kritische Vierteljahresschrift für Gesetzgebung und Rechtswissenschaft 25 (1932), 250-296; 27 (1934), 1846.

47 Artur Steinwenter, Das Recht der koptischen Urkunden. Handbuch der Altertumswissenschaft, Rechtsgeschichte des Altertums, 4. Teil: Das Recht der Papyri, 2. Bd., München 1955. 
3. Die im thebanischen Raum unter der Bezeichnung Epitrope ausgestellten Urkunden wurden bisher nicht klar als das gesehen und gewürdigt, was sie eigentlich sind: Pachturkunden. Charakteristisch für diese Unsicherheit ist die Terminologie Walter Tills, des seinerzeit besten Kenners koptischer Rechtsurkunden: In Anlehnung an die griechische Selbstbezeichnung dieser Urkunden єпıтропн und ihre Eingangformel †епıтрепє NaK єTрєкхо, , ich (beauftrage dich/ermächtige dich/gestatte dir), daß du säst' nannte Till diese Texte ,Ackerbestellungen' oder ,Ackerbestellaufträge‘. In O.Theb. 6, einem Fragment eben solch einer Urkunde, dessen Eingangsformel mit dem Verb غ̇лıре́́лєıv und Schluß mit der Urkundenbezeichnung єпıтропн jedoch nicht erhalten sind, vermutete Till hingegen eine „Ackerbestellung oder Verpachtung“, da ,außer dem Ausdruck ,Aussaat “ ... nur erkennbar" sei, „,daß der Urkundendestinatar Pachtzins ( $\pi$ ók $\tau$ ov) zahlen soll."48 Ein ebenfalls ohne Anfang und Schluß erhaltenes Fragment einer Epitrope, BKU I 75, bezeichnete Till rundweg als „Feldverpachtung“49. Offenkundig ließ sich Till

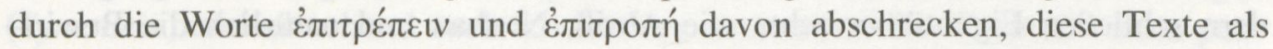
Pachturkunden anzusprechen. Auch Artur Steinwenter 1955, 40f. beschrieb umständlich den Doppelcharakter der Epitrope als Arbeitsvertrag und Pachtverhältnis, wobei er durch ein eingeklammertes Ausrufungszeichen seine Verwunderung darüber zum Ausdruck brachte, daß bei dieser Art von „Aufträgen“ dem Auftraggeber ein Entgelt gezahlt wurde. Weniger irreführend ist die von Crum gewählte Bezeichnung ,authorization to sow', die auch Arthur Schiller benutzte ${ }^{50}$. Unten werde ich noch einmal darauf zu sprechen kommen.

4. Aus demselben Grund wurden zwei als Epitrope bezeichnete Papyrusurkunden, P.KRU 57 und 59, bisher nicht als antichretische Pachturkunden erkannt, in denen ein in der Schuld seines Pächters stehender Verpächter zusagt, sein eigenes Feld zu bestellen, um den Gläubiger/Pächter mit einer ohne dessen Arbeitsaufwand erzeugten Ernte zu entschädigen.

5. Schließlich sind einzelne Dokumente noch grundsätzlicher verkannt worden. So ist die späteste datierte koptische Pachturkunde, P.Lond. Copt. I 487 aus dem Jahr A.H. 287 = A.D. 900, von Crum 1905 als ein inhaltlich obskurer Brief ediert worden. ${ }^{51}$ Crum konnte die entscheidende Wendung nicht deuten: $\alpha=1-T 1 N \lambda=K$

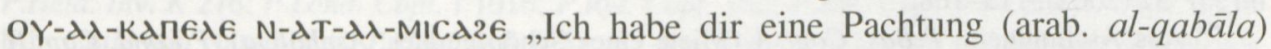
ohne (arab. $b i-l \bar{a}-=$ kopt. N-גT-) Vermessung (arab. al-misāḥa) gegeben“. Hier ist nämlich die Bezeichnung einer zu Crums Zeit noch unbekannten arabischen Pachtart ins Koptische teils entlehnt, teils übersetzt worden. ${ }^{52}$

48 Walter C. Till, Die koptischen Rechtsurkunden aus Theben. SÖAW, Phil.-hist. K1., 244. Bd., 3. Abhg. Wien 1964, 230.

49 Till, Die koptischen Rechtsurkunden aus Theben (s.o., Fn. 48), 18.

50 A. Arthur Schiller, „Koptisches Recht. Eine Studie auf Grund der Quellen und Abhandlungen“, in: Kritische Vierteljahresschrift für Gesetzgebung und Rechtswissenschaft 25 (1932), 281f.: „Ermächtigung“.

${ }^{51}$ Walter C. Crum, Catalogue of the Coptic Mss, in the British Museum, London 1905.

52 Tonio Sebastian Richter, ,Spätkoptische Rechtsurkunden neu bearbeitet (III): P.Lond. Copt. I 487 - Arabische Pacht in koptischem Gewand“, in: JJP 33 (2003), 213-230. 
Im folgenden möchte ich einen ersten Überblick über die koptischen Quellen zur Pacht geben.

Koptische Miet- und Pachturkunden sind sehr mannigfaltig. Zur Miete, also gegen Zahlung von a)6op, wurden zumeist Gebäude zu einfacher Nutzung überlassen. Die zwölf erhaltenen koptische Urkunden über Miete von Häusern und Hausteilen sind vor einigen Jahren neuediert und kommentiert worden. ${ }^{53}$ Für ()6op wurden auch Geräte wie Wasserschöpfmaschinen oder ein Webstuhl vermietet. ${ }^{54}$ Einmal wird Boden - das häufigste Pachtobjekt, vermietet ${ }^{55}$ Eine Partei möchte eine an die Wand ihres Hauses angrenzende Fläche in ihren Besitz bringen; das Motiv der Fruchtziehung fehlt also. Andererseits konnten Häuser nicht nur vermietet, sondern verpachtet werden. Namentlich Erbpachturkunden haben häufig Gebäude zum Objekt. ${ }^{56}$ Der Unterschied zur Hausmiete besteht erwartungsgemäß in den Nutzungsrechten. Diese erschöpfen sich bei der Pacht nicht im Wohnrecht und damit untrennbaren Rechtstiteln wie Eingang und Ausgang, sondern schließen Eigentümerrechte wie Abriß, Neubau und natürlich die Bewirtschaftung des Gebäudes, z.B. wiederum durch Vermietung, ein.

Die meisten Pachturkunden betreffen aber, wie gesagt, Bodenpacht. Wir kennen vier koptische Urkunden über Bodenpacht aus dem Faijum. Eine einzelne Urkunde aus Ehnas, ${ }^{57}$ eine vom Pächter ausgestellte Misthosis, unterscheidet sich in vielerlei Hinsicht von drei Urkunden aus Medinet el-Faijum, ${ }^{58}$ die von den Verpächtern ausgestellt sind. In diesen drei Urkunden steht regelmäßig die Bezeichnung ()ом für ,Pachtzins'. Dieses lokale Rechtswort kommt bereits in den demotischen Pachturkunden des Faijum vor, während in demotischen Pachturkunden aus Oberägypten der altertümliche Ausdruck $h w$-` $h w t$ gebraucht wurde, der in der Terminologie koptischer Pachturkunden durch griechische Lehnwörter ersetzt ist. $^{59}$

Die koptischen Misthosis-Urkunden aus der Region von Aschmunein und Antinoupolis $^{60}$ gebrauchen grundsätzlich den Ausdruck фopoc, genau wie die

53 Tonio Sebastian Richter, „Koptische Mietverträge über Gebäude und Teile von Gebäuden“, in: JJP 32 (2002), 113-168.

54 Aus Aschmunein: P.Bas. Copt. 1 (Miete eines Schöpfgerätes, Asphaleia); P.Lond. Copt. I 1020 (Miete eines Männer-Webstuhls, Misthosis). Aus Theben: O.Crum 140 (Miete eines Schöpfgerätes).

55 P.Ryl. Copt. 165 (aus Aschmunein).

${ }^{56}$ Vgl. neben den beiden Emphyteusis-Urkunden CPR IV 128 und P.Lond. Copt. I 1015 auch die Quittungen über die Zahlung von Erbpachtzins (pakton) für die Erbpacht von Gebäuden: $C P R$ IV 147; CPR IV 152; CPR IV 153; BL Or 6201 A 66; BL Or 6201 A 22a; BL Or 6201 A 103; P.Beatty Copt. inv. 2177.7; BL Or 6201 A 22b; BL Or 6201 A 68 und BL Or 6201 A 23.

${ }^{57}$ CPR IV 120.

${ }^{58}$ CPR IV 126; CPR IV 127; CPR IV 129a = CPR II 131.

59 Vgl. Tonio Sebastian Richter, „Alte Isoglossen im Rechtswortschatz koptischer Urkunden“, Lingua Aegyptia 10, 389-399.

${ }^{60}$ Mit Festpacht in Geld: $B K U$ III 347; BKU III 348; CPR IV 117; CPR IV 118; P.Lond. Copt. I 1022; P.Mon. Apollo 26 (= P.HermitageCopt. 3); P.Ryl. Copt. 163; P.Ryl. Copt. 173. Mit pauschalem Naturalzins: $C P R$ IV 119; $C P R$ IV 122; CPR IV 123; CPR II 139 (= CPR IV 129b); 
griechischen Pachturkunden dieser Region, deren Formular sie fast wortgetreu nachgebaut sind. Diese Misthosis-Urkunden unterscheiden sich voneinander durch verschiedene Absprachen über zu leistende Arbeiten, die Investition von Saatgut und Unkosten für Lohnarbeiter, Vieh und Arbeitsgerät sowie über die Art und Höhe des Pachtzinses. Einige Urkunden enthalten Nichtkündigungs-Klauseln, in denen sich der Pächter bis zu zehn Jahren an sein Pachtgut bindet. Dabei verzichtet der Pächter ausdrücklich auf die Wirkung eines Gesetzes, das Pächtern in permanenten Pachtverhältnissen die Kündigung innerhalb des ersten Jahres zubilligt. Einige Urkunden enthalten Abrochia-Klauseln, in denen das Risiko von Ernteausfall durch Trockenheit geregelt ist. Es tritt dann eine Minderung der Pächterabgabe in Kraft (remissio mercedis), indem ein alternativer Zahlungsmodus für den Pachtzins vereinbart wird, der das Risiko einigermaßen symmetrisch auf Pächter und Verpächter verteilt. Die vom Pächter zu leistenden Abgaben setzten sich aus dem Pachtzins, einigen für die byzantinische Zeit typischen Sonderleistungen (Synetheia) und manchmal auch aus Steuern zusammen. Der Pachtzins kann pauschal in Geld (xpycıkос форос u.ä.) oder in einer festen oder quotierten Menge von Ackerfrüchten bestehen. In zwei sehr ausführlichen Urkunden ${ }^{61}$ über Felder und Gärten wird ein aus einem Geldbetrag und einer Teilpachtquote der Obsternte kombinierter Pachtzins vereinbart. Die steuerliche Zuständigkeit wird zumeist nicht erwähnt, und dies bedeutet wohl, daß sie formal beim Verpächter lag.

Nur aus dem Raum von Aschmunein sind koptische Erbpachtverträge bekannt, ${ }^{62}$ deren Pachtzins hier Pakton oder Emphyteuma genannt wurde. ${ }^{63}$ Ihre Besonderheit gegenüber den meisten koptischen Urkunden besteht darin, daß sie, bei subjektiver oder objektiver Stilisierung, grundsätzlich bilateral abgefasst sind und doppelt ausgefertigt wurden. Die Erbpacht-Verträge und Erbpachtzins-Quittungen zeigen in der Rolle der Verpächter fast nie einzelne Privatpersonen, sondern Erbengemeinschaften, eine Dorfgemeinschaft (Кoเvóv) sowie am häufigsten das Dikaion eines Klosters, einer Kirche oder einer Laienvereinigung (Philoponeion). Die typischerweise mit Emphyteuse verbundene ökonomische Strategie,

P.Heid. Inv. K 276; P.Lond. Copt. I 1016; P.Ryl. Copt. 162; P.Ryl. Copt. 164; P.Ryl. Copt. 170 ?. Mit quotiertem Naturalzins: P.Heid. Inv. K 38; P.Lond. Copt. I 1073. Mit gemischtem System aus Natural- und Geldzins und Teilpachtelementen: P.Ryl. Copt. 158; P.Ryl. Copt. 159; weitere Fragmente von Misthosis-Urkunden, wobei teilweise unklar, ob Miete oder Pacht: $C P R$ IV 121; $C P R$ IV 124; CPR IV 129c; CPR IV 129d; CPR IV 129e; CPR IV 129f.; P.Hermitage Copt. 2; P.Laur. Copt. 193; P.Lond. Copt. I 1019; P.Lond. Copt. I 1021; P.Lond. Copt. I 1067; P.Ryl. Copt. 161; P.Ryl. Copt. 166; P.Ryl. Copt. 167; P.Ryl. Copt. 168; zur letzten Kategorie zählen auch die hier vorgestellten Fragmente von Misthosis-Urkunden aus P.Yale inv. 1804.

${ }^{61}$ P.Ryl. Copt. 158; P.Ryl. Copt. 159.

62 Erbpacht von Häusern: CPR IV 128; P.Lond. Copt. I 1015. Erbpacht von Garten- und Ackergrundstücken: P.Lond. Copt. I 1013; P.Ryl. Copt. 174; P.Ryl. Copt. 176. Weitere Fragmente von Erbpacht-Urkunden: P.Lond. Copt. I 1014; P.Ryl. Copt. 175.

63 Quittungen über Pakton aus dem Gebiet von Aschmunein, wie CPR IV 146-53, P.Lond. Copt. I 1027 und 1056, P.Ryl. Copt. 179 und 180 und SB Kopt. I 243, dürften sich dementsprechend auf Erbpachtverhältnisse beziehen, wie es etwa in CPR IV 146, 147 und 148 und in $S B$ Kopt. I 243 auch explizit gesagt wird. 
mittels der Pacht aus Grundbesitztümern Rendite zu erzielen und so Anlagekapital zu verflüssigen, zeigt sich auch darin, daß der Pachtzins hier fast immer in Geld zu zahlen ist.

Die bisher einzige bekannte koptische Pachturkunde aus Aphrodito, P.BL inv. 2849 , ist noch unpubliziert. ${ }^{64}$

Die meisten Pachturkunden aus dem thebanischen Raum heißen, wie gesagt, Epitrope. Nur zwei Misthosis-Urkunden sind bekannt, ${ }^{65}$ die, wie immer bei diesem Typus, vom Pächter ausgestellt sind. Die Epitrope-Urkunden dagegen sind stets vom Verpächter ausgestellt, ${ }^{66}$ und sie fokussieren die landwirtschaftliche Arbeit des Pächters in der einzigen Formulierung єтрєкхо „dass du besäst“. In diesen beiden Merkmalen ähneln sie späteren arabischen Pachturkunden, die ebenfalls vom Verpächter ausgefertigt sind und deren Klausel über landwirtschaftliche Arbeit in gleicher Weise als pars pro toto die Feldbestellung nennt. Weitere Klauseln der Epitrope betreffen die Pächterabgaben - Teilpacht im Halbe-HalbeProporz ist ein gängiger Modus - und die Verteilung der Investitionen. Es ist auffallend, daß die thebanischen Pächter oft für Zugvieh, Lohnarbeiter und Unkosten sowie für einen Teil des Saatgutes aufkommen sollten: Schmälert das auch ihren

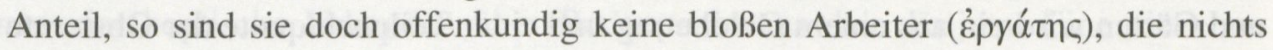
als ihre eigene Arbeitskraft zu verdingen hätten, sondern leistungsfähige Partner der Verpächter.

Meines Wissens sind bisher nur drei Epitrope-Urkunden in griechischer Sprache bekannt geworden ${ }^{67}$ welchen ein mehrfaches an koptischen Dokumenten dieses Typs und dieser Bezeichnung gegenübersteht - eine ungewöhnliche Konstellation, die so kaum irgendwo anders als im koptophonen Djême denkbar ist. Viel-

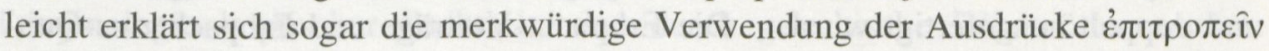
bzw. غ̇лıтроли́ aus dem ägyptischsprachigen Substrat der lokalen Pacht-Terminologie. Ich möchte hier wenigstens eine Vermutung über die Bedeutung und die Etymologie dieser Termini in Theben wagen: Der demotische Ausdruck für, ver-

64 Vgl. dazu einstweilen Lucia Papini, ,Notes on the Formulary of some Coptic Documentary Papyri from Middle Egypt“, BSAC 25 (1983), 83-89; Dies., „Annotazioni sul formulario giuridico di documenti copti del VI secolo", in: Atti del XVII congresso internazionale di Papirologia, Napoli 1984, 767-776; Dies., „A Lease of Land from Aphroditopolis“, in: W. Godlewski (Hrsg.), Coptic Studies, Acts on the Third International Congress of Coptic Studies, Warsaw, 20-25 August 1984, Varsovie 1990, 299-302. Die Edition der Urkunde, an welcher Anne Boud'hors (Paris), Hans Förster (Wien), Jean-Luc Fournet (Paris), Fritz Mitthof und der Vf. dieses Artikels mitarbeiten, ist in Vorbereitung.

${ }^{65}$ BKU I 82; O.CrumVC 33 (aus El-Tod).

${ }^{66}$ Mit Festpacht in Geld: $B K U$ I 79. Mit Festpacht in Naturalien: $B K U$ I $64 ; B K U$ I $75 ; B K U$ I 95; O.Crum 206; O.Engelbach 3; O.Brit. Mus. Copt. I 35/4; O.CrumVC 28. Mit Festpacht aus Geld und Naturalien gemischt: O.Crum 307. Mit Festpacht (Details unklar: BKU I 40; O.Theb. 6. Mit Mischzins aus Geld und erfolgsabhängiger, quotierter Ertragsabgabe: O.Crum 139; O.Crum Ad. 26. Mit Ertragsteilung: BKU I 48 (bilateral); O.Crum 138; O.Crum 482; O.Vind. Copt. 42 (= O.CrumST 37); O.CrumST 38; O.CrumST 39; O.Medin. Habu Copt. 81.

${ }^{67}$ O.Wilcken 1224, PSI III 279 und O.Qurnat Mar'y inv. 249 (ed. Gascou, BIFAO 1999). 
pachten ' ist $s h n .^{68} \mathrm{Er}$ ist letztmals in den spätesten demotischen Rechtsurkunden überhaupt belegt, in thebanischen Ostraka des 2. Jhs. bis frühen 3. Jhs. n.Chr., in denen Priester einander Tempelliturgien verpachteten. ${ }^{69}$ Dieses Rechtswort hat ausschließlich und ausgerechnet in Theben überdauert. Koptische Rechtsurkunden aus Djême verwenden ein Nomen carne in der Bedeutung "Miet-" bzw. ,Pachturkunde'.$^{70}$ Die eigentliche Bedeutung des demotischen Verbs shn reicht indessen von , beauftragen ${ }^{71}$ bis hin zu ,überlassen, anvertrauen', ${ }^{72}$, confier', ${ }^{73}$, to hand over to a persons care ${ }^{64}$. Sie deckt sich also weitgehend mit dem

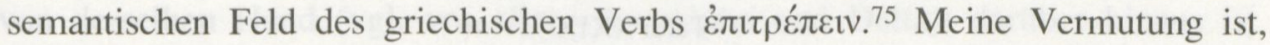

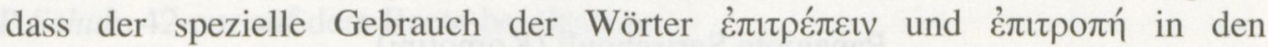
thebanischen Pachturkunden durch eine Bedeutungsentlehnung vom Verb shn , anvertrauen etc.' bzw. dem zugehörigen Nomen shn ,Pachturkunde' inspiriert worden ist.

${ }^{68}$ Vgl. Felber, Demotische Ackerpachtverträge der Ptolemäerzeit (s.o., Fn. 39), $116 \mathrm{ff}$.

${ }^{69}$ Ediert von Herbert Thompson, Theban Ostraca, London, Oxford 1913.

70 Vgl. Richter, „Alte Isoglossen im Rechtswortschatz koptischer Urkunden“ (s.o., Fn. 59), 394-395.

${ }^{71}$ Erichsen, Demotisches Glossar, Kopenhagen 1954, 446.

${ }^{72}$ Felber, Demotische Ackerpachtverträge der Ptolemäerzeit (s.o., Fn. 39), $116 \mathrm{ff}$.

73 P.W. Pestman, Recueil de textes démotiques et bilingues. 3 vol.s. Leiden 1977, vol. II 102.

${ }^{74}$ P.W. Pestman, The Archive of the Theban Coachytes (Second Century B.C.). A Survey of Greek Papyri Contained in the Archive. Studia Demotica 2, Leuven 1993, 150, Anm. c.

${ }^{75}$ Preisigke, $W b$ I 582/3: , auftragen, anbefehlen, gestatten, zulassen, erlauben'; $L S J 667 / 8$ : ,to commit, to entrust to, to rely upon, to leave to, to yield up, to permitt, to command". 